\title{
Structural basis of template strand deoxyuridine promoter recognition by a viral RNA polymerase
}

\section{Petr Leiman ( $\nabla$ pgleiman@utmb.edu )}

University of Texas Medical Branch https://orcid.org/0000-0002-9091-0918

\section{Alec Fraser}

University of Texas Medical Branch

\section{Maria Sokolova}

Skolkovo Institute of Science and Technology https://orcid.org/0000-0001-7251-6952

\section{Arina Drobysheva}

Skolkovo Institute of Science and Technology

\section{Julia Gordeeva}

Skolkovo Institute of Science and Technology

\section{Sergei Borukhov}

Rowan University School of Osteopathic Medicine at Stratford https://orcid.org/0000-0002-3517-3003

\section{AlphaFold Team}

DeepMind Technologies Limited

\section{Konstantin Severinov}

Rutgers University https://orcid.org/0000-0001-9706-450X

\section{Article}

Keywords: RNA polymerases, transcription start site, virus evolution

Posted Date: November 4th, 2021

DOl: https://doi.org/10.21203/rs.3.rs-887640/v1

License: (c) (1) This work is licensed under a Creative Commons Attribution 4.0 International License. Read Full License

Version of Record: A version of this preprint was published at Nature Communications on June 20th, 2022. See the published version at https://doi.org/10.1038/s41467-022-31214-6. 


\title{
Structural basis of template strand deoxyuridine promoter recognition by a viral RNA polymerase
}

\author{
Alec Fraser ${ }^{1, \#}$, Maria L. Sokolova ${ }^{2, \#, *}$, Arina V. Drobysheva ${ }^{2}$, Julia V. Gordeeva ${ }^{2}$, Sergei Borukhov ${ }^{3}$, \\ AlphaFold team ${ }^{4}$, Konstantin V. Severinov ${ }^{5,6, *}$, Petr G. Leiman ${ }^{1 *}$
}

${ }^{1}$ Department of Biochemistry and Molecular Biology, Sealy Center for Structural Biology and Molecular Biophysics, University of Texas Medical Branch, Galveston, TX 77555-0647, USA

${ }^{2}$ Center of Life Sciences, Skolkovo Institute of Science and Technology, Moscow, 121205, Russia

${ }^{3}$ Department of Cell Biology and Neuroscience, Rowan University School of Osteopathic Medicine at Stratford, Stratford, NJ 08084-1489, USA

${ }^{4}$ DeepMind Technologies Limited, 5 New Street Square, London EC4A 3TW

${ }^{5}$ Institute of Molecular Genetics, Russian Academy of Sciences, Moscow, 123182, Russia

${ }^{6}$ Waksman Institute for Microbiology, Rutgers, The State University of New Jersey, Piscataway, NJ 08854, USA

\# Contributed equally

* Corresponding authors

E-mail: pgleiman@utmb.edu, maria.sokolova@skolkovotech.ru, severik@waksman.rutgers.edu 


\begin{abstract}
Recognition of promoters in bacterial RNA polymerases (RNAPs) is controlled by their sigma subunits that first bind to a double-stranded DNA element $\sim 35$ to $\sim 14$ nucleotides upstream of the transcription start site (TSS) and then capture several bases of the non-template strand $\sim 10$ nucleotides upstream of the TSS. Here, we shift this paradigm and explain the mechanism by which the phage AR9 non-virion RNAP (nvRNAP), a bacterial RNAP homolog, recognizes a -10 promoter element in the template strand directly. Together, the AR9 nvRNAP sigma-like subunit, the enzyme core, and the template strand itself form two nucleotide base-accepting pockets whose shapes dictate the AR9 nvRNAP's deoxyuridine promoter requirement. A single amino acid substitution in the promoter specificity subunit allows one of these pockets to accept a thymine base thus expanding the enzyme's promoter consensus. Our work demonstrates the extent to which viruses can evolve host-derived multisubunit enzymes to control host takeover.
\end{abstract}




\section{Introduction}

Bacillus subtilis “jumbo" bacteriophage AR9 encodes two distinct multisubunit DNA-dependent RNA polymerases (RNAPs), allowing for the transcription of viral genes to proceed independently of the host $\mathrm{RNAP}^{1-4}$. The virion-packaged RNAP (vRNAP) is delivered into the host cell together with phage DNA at the onset of infection. The vRNAP then transcribes early phage genes, including those of the second, non-virion RNAP (nvRNAP). The nvRNAP transcribes late genes, including those coding for the vRNAP, which is packaged into progeny phage particles together with phage DNA. Notably, the double stranded (ds) genomic DNA of AR9 contains uracils instead of thymines throughout ${ }^{1,2}$.

The catalytically active AR9 nvRNAP core enzyme consists of four proteins that, when pairwise concatenated, show about $20 \%$ sequence identity and cover the entire lengths of the universally conserved $\beta$ and $\beta^{\prime}$ subunits of bacterial RNAPs (Fig. 1a) $)^{3}$. Promoter-specific transcription is performed by a five-subunit holoenzyme that in addition to the nvRNAP core contains the product of AR9 gene 226 (gp226) ${ }^{3}$. Gp226 shows no discernible sequence similarity to bacterial RNAP $\sigma$ subunits, which mediate the recognition of promoters in bacteria, or any known transcription factor. Close orthologs of gp226 are found in the genomes of other jumbo phages that have been demonstrated or are presumed to contain uracil in their genomic $\mathrm{DNA}^{5,6}$.

Unlike bacterial RNAPs ${ }^{7}$, the AR9 nvRNAP holoenzyme recognizes promoters in the template strand of dsDNA and is capable of promoter-specific transcription initiation on single stranded (ss) $\mathrm{DNA}^{3}$. The AR9 nvRNAP template-strand promoter consensus $3^{\prime}-{ }^{-11} \mathrm{UUGU}^{-8}-\mathrm{N}_{6}-\mathrm{AU}^{+1}-5^{\prime}$ (where $\mathrm{N}$ is any nucleotide and the transcription start site (TSS) coordinate is +1 ) contains a four base-long motif centered about 10 nucleotides upstream of the TSS and two nucleotides at the TSS (Fig. 1b). Promoters with thymines in the $-11^{\text {th }}$ and $-10^{\text {th }}$ positions are inactive, suggesting that the $\mathrm{C} 5$ position of the uracil's pyrimidine ring, which carries a methyl group in the thymine, plays a critical role in promoter recognition 
(Fig. 1c). Despite possessing a short promoter consensus element, the AR9 nvRNAP holoenzyme protects an extensive region of DNA flanking the TSS (position -35 to +20 in the template strand and positions -29 to +17 in the non-template strand) from DNase I attack, implying additional contacts with $\mathrm{DNA}^{3}$.

To understand the uracil-specific, template strand-dependent promoter recognition mechanism of the AR9 nvRNAP, we determined the structure of this enzyme by X-ray crystallography and cryoelectron microscopy (cryo-EM) in three states - the core, holoenzyme, and holoenzyme in complex with a 3'-overhang dsDNA oligonucleotide that mimicked the downstream half of the transcription bubble (historically called a "forked" or "fork" template) - and complemented this structural information by discriminative in vitro transcription assays. In its ss part, the forked oligonucleotide contained the P077 promoter of the AR9 late gene 076 which encodes a highly expressed virion protein of unknown function $^{2}$. 


\section{Results}

\section{Brief description of structural data}

We have crystallized the AR9 nvRNAP core (Fig. 1a) in two different crystal forms that had a "standard" and large unit cell and contained two and eight nvRNAP core molecules in their asymmetric units, respectively. The standard and large unit cell crystals diffracted X-rays to $3.30 \AA$ and $3.79 \AA$ resolutions, respectively (Supplementary Table 1). The holoenzyme (Fig. 1a) failed to produce crystals, so we used cryo-EM to calculate its electron density map to a resolution of $4.4 \AA$ (Supplementary Table 2).

The structure of the promoter complex has been determined by both X-ray crystallography and cryo-EM to resolutions of $3.38 \AA$ and $3.80 \AA$, respectively (Supplementary Table 1, 2). Two different crystal forms of the promoter complex were obtained. Both forms displayed one common crystal packing property that is described and illustrated in detail below. The conformations of the enzyme in both crystal forms were similar but the quality of the DNA electron density differed. The promoter complex dataset with a higher fraction of ordered DNA was used for analysis here.

We first obtained an interpretable electron density for the core enzyme by a procedure described in the Methods section. The core displayed a significant amount of disorder preventing the completion of the model. A partial model of the core was used to interpret the cryo-EM and X-ray electron densities of the promoter complex. The latter was ordered almost in its entirety which allowed us to build nearly complete atomic models for all five proteins (Fig. 1a) and for a large fraction of DNA comprising the complex. Upon refinement against the experimental data, the cryo-EM and X-ray structures were very similar and could be superimposed with a root mean square deviation (RMSD) of $1.84 \AA$ between 2,563 or $97 \%$ of all $\mathrm{C} \alpha$ atoms of the holoenzyme. The promoter complex X-ray structure contained a larger fraction of ordered DNA and had a higher resolution than the cryo-EM map. Consequently, only the X- 
ray model of the promoter complex has been fully refined and deposited to the Protein Data Bank (PDB) whereas the cryo-EM density of the same complex has been deposited to the Electron Microscopy Data Bank (EMDB) without accompanying model coordinates (Supplementary Table 1, 2). The resolution of the cryo-EM map of the holoenzyme (4.4 $\AA$ ) did not permit building a reliable atomic model, so it has been deposited to the EMDB also without an atomic model (Supplementary Table 2). Finally, the model of the AR9 nvRNAP core, which had been refined against the $3.30 \AA$ resolution X-ray data of the standard cell dataset, has been deposited to the PDB (Supplementary Table 1).

Being the most complete, the $3.38 \AA$ resolution X-ray structure of the AR9 nvRNAP promoter complex is used below in the description of various structural features of the enzyme such as the conformation of the active site and the folds of various domains. Other datasets are referenced according to their trivial names (e.g. the core or the holoenzyme) and are used in the interpretation according to the limit of their accuracy - e.g. atomic coordinates for the core and cryo-EM map for the holoenzyme .

\section{Structural similarities and differences between the AR9 nvRNAP and bacterial RNAPs}

In the most populous class of the cryo-EM reconstruction and in both available crystal forms of the promoter complex, the enzyme bound not one but two copies of the P077 promoter-containing forked oligonucleotide - a downstream copy (as designed) and an upstream one (fortuitously) - resulting in a superstructure that resembled the complete transcription bubble found in open complexes formed by other RNAPs (Fig. 2a, 2b). Moreover, in crystallo the nvRNAP molecules and the oligonucleotides formed a train in which the upstream and downstream oligonucleotides belonging to two neighboring unit cells pi-stacked and formed a continuous double helix (Supplementary Fig. 1).

The overall structure of the AR9 nvRNAP is a trimmed down version of a bacterial crab clawshaped RNAP (Fig. 2a, Fig. 3). No domain compensates for the absence of $\alpha$ and $\omega$ subunits that are present in all bacterial, eukaryotic, and archaeal enzymes. As a result, the AR9 nvRNAP claw is smaller 
and has a boxier appearance than that of its cellular counterparts. In bacterial enzymes, the $\alpha$ subunit dimer serves as a platform for the assembly of the $\beta$ and $\beta^{\prime}$ subunits $^{8-10}$. In the AR9 nvRNAP structure, the split site of the $\beta^{\prime}$ subunit is spatially close to the putative $\beta^{\prime}-\alpha^{I I}$ interface (Fig. 3 ), which suggests that this location likely represents a critical point for the formation of tertiary and quaternary structure.

Inside the catalytic cleft, the AR9 nvRNAP core contains all of the structural elements required for catalysis, stabilization of the open promoter complex, and promoter clearance found in multisubunit RNAPs $^{9,11}$, except for the $\beta^{\prime}$ rudder (Fig. 2c, 2d, Fig. 3). The $\beta^{\prime}$ rudder is a twisted $\beta$-hairpin that is present in all known RNAPs. It extends from one of the $\beta^{\prime}$ clamp $\alpha$-helices and interacts with the RNADNA hybrid near the active site ${ }^{9}$. In bacterial RNAPs, deletion of the $\beta^{\prime}$ rudder impairs promoter opening and destabilizes the elongation complex but does not affect the efficiency of transcription termination or the length of the RNA-DNA hybrid ${ }^{12}$. The elongation complex of the AR9 nvRNAP must be stabilized by a different mechanism. The conformation of the ${ }^{370}{ }^{2}$ YDGD $^{374}$ catalytic motif of the AR9 nvRNAP, which is located near the $\mathrm{C}$ terminus of the $\beta^{\prime} \mathrm{N}$ subunit gp270, is similar to that found in other RNAPs. The side chains of the three conserved aspartates are poised to bind a $\mathrm{Mg}^{2+}$ ion that is universally conserved in all nucleotidyltransferases, albeit the resolution of X-ray and cryo-EM data is insufficient for resolving it (Fig. 2c, 2d, Fig. 3a). The electron density of DNA at the TSS is also relatively poor and, as such, a structural basis for the conservation of the TSS and the nucleotide preceding it (Fig. 1b) cannot be determined.

Similar to the E. coli RNAP $^{13}$, the AR9 nvRNAP contains an insertion domain (residues 400508 of $\beta^{\prime} \mathrm{C}$ gp 154) in the trigger loop (Fig. 2a, Fig. 3). The trigger loop undergoes major conformational changes during the catalytic nucleotide addition cycle and template translocation ${ }^{9,11}$, and the presence of an insertion domain in the E. coli system has not been fully reconciled with these transformations ${ }^{14}$. The fold of the AR9 nvRNAP insertion domain is different from that of the E. coli RNAP and, in fact, to any 
protein in the PDB. Its sequence is also unique and found only in nvRNAPs of other jumbo phages ${ }^{5,6}$. Its position in the structure of the nvRNAP core is also different from that of the E. coli RNAP. In the AR9 nvRNAP core structure, the insertion domain is located roughly in-between the $\beta$ and $\beta^{\prime}$ pincers where it partially obstructs the downstream DNA channel (Fig. 4a). Furthermore, it carries a strong negative charge on its DNA-facing surface suggesting that it interacts with the downstream dsDNA in a sequence independent manner (Fig. 4b). Out of 10 independent copies of the AR9 nvRNAP core molecules belonging to two different crystal forms (standard and large unit cell crystals, Supplementary Table 1), the insertion domain is ordered in only a singular instance. In the cryo-EM structure of DNA template-free holoenzyme, this domain is fully disordered (Fig. 5a, 5b). Considering the intrinsic propensity of this domain to large motions, it may participate in translocation by sliding on the DNA and exerting a force on the trigger loop.

\section{The structure of promoter-specificity subunit gp226}

The AR9 nvRNAP promoter-specificity subunit gp226 consists of two globular domains - a larger N-terminal domain (NTD, residues 1-264) and a smaller C-terminal domain (CTD, residues 295464) - connected by a linker (Fig. 2a, Fig. 6a).

Gp226 interacts with the nvRNAP core in a manner resembling that of bacterial $\sigma$ factors ${ }^{7,11,15,16}$, and all elements that come in contact with the body of the enzyme have structural counterparts in bacterial $\sigma$ factors. Residues 184-264 of gp226 fold into a $\sigma_{2}$-like domain (Fig. 6a), which interacts in a sequence specific manner with the non-template strand of the -10 promoter element in bacterial $\sigma$ factors and comprises their most conserved part ${ }^{17-19}$ (Fig. 6b). Gp226 residues 265-294 form a $\sigma$ finger-like structure that invades the catalytic cleft and forms an augmented $\beta$-sheet with the $\beta^{\prime}$ lid (Fig. 2c, Fig. 6a). Gp226 residues $295-316$ comprise an $\alpha$-helix that matches the $N$-terminal $\alpha$-helix of the $\sigma_{4}$ domain (Fig. 6a, 6b). 
The most similar $\sigma$ factor with a known structure, the $E$. coli $\sigma^{\mathrm{E}}$, displays a C $\alpha$-C $\alpha$ RMSD of 4.2 $\AA$ and a sequence identity of $9.6 \%$ when superimposed onto residues $184-316$ of gp226 which comprise its $\sigma_{2}$, finger- and $\sigma_{4}$-like elements. Thus, the $\sigma$-like part of gp226 spans a contiguous region of 133 residues and is nearly equal in size to the entire $\sigma^{\mathrm{E}}$ structure (Fig. 6a, 6b). Considering the structural similarity of gp226 to the conserved part of bacterial $\sigma$ factors, their similar functions, and the similar manner by which they interact with the template DNA (the structure of the transcription bubble), it follows that gp226 is a homolog of bacterial $\sigma$ factors, despite their low sequence identity. The peripheral parts of the gp226 NTD and CTD have been replaced with new folds but all elements that interact with the body of the enzyme and with DNA (albeit with some modifications for the latter) have been retained.

A Protein Data Bank-wide search ${ }^{20}$ for folds resembling that of the gp226 NTD and CTD resulted in a single definitive match. The CTD of gp68, a subunit of the phage phiKZ non-virion RNAP which is required for both promoter recognition and transcription elongation ${ }^{21,22}$, can be superimposed onto the gp226 CTD with an RMSD of $3.2 \AA$ for 142 equivalent $C \alpha$ atoms (out of 173) and a sequence identity of 11\% (Supplementary Fig. 2a). As the rest of the gp68 structure (residues 1-303) is disordered, we used AlphaFold ${ }^{23}$ Colab to model it. The local distance difference test ${ }^{24}$ of this model for residues 1-277 was 85.3, indicating a very high level of confidence (Supplementary Fig. 2b). This model can be superimposed onto the gp226 NTD with an RMSD of $3.0 \AA$ for 198 equivalent C $\alpha$ atoms (out of 277) and a sequence identity of 8.6\% (Supplementary Fig. 2a). Thus, even though gp226 is as divergent from gp68 sequence-wise as it is from bacterial $\sigma$ factors, their similarities in structure and location within the RNAP holoenzyme complex suggest that all these proteins have a common ancestor.

\section{Structural adaptations of gp226 required for template strand promoter recognition}

The most conserved parts of bacterial $\sigma$ factors, the $\sigma_{2}$ and $\sigma$ finger elements, are also present in gp226 (Fig. 6). With small adaptations, these elements are responsible for the unique mode of promoter 
recognition through template DNA strand. A tight turn connecting helices 2.1 and 2.2 in bacterial $\sigma$ factors is replaced by a short $\alpha$-helix in gp226 (residues 205-214). This 2.1 bis helix creates a bridge linking the two pincers of the AR9 nvRNAP claw that, together with the $\beta \mathrm{N}$ gp105 subunit, comprises a binding site for the promoter in the template strand of DNA (Fig. 6a). The gp226 finger forms an augmented $\beta$ sheet with the $\beta^{\prime}$ lid (residues $161-177$ of $\beta^{\prime} \mathrm{N}$ gp270) such that the $\beta^{\prime}$ lid reaches the -8 position uracil base of the $3^{\prime}-{ }^{-11} \mathrm{UUGU}^{-8}-5^{\prime}$ promoter motif and tucks it in against the 2.1 bix helix (Fig. $\mathbf{2 c}, \mathbf{2 d})$. The $\beta^{\prime}$ lid of AR9 nvRNAP is three residues longer than its bacterial counterpart, which further enhances and facilitates this interaction (Fig. 2c).

\section{Promoter DNA structure and the design of a T-specific enzyme}

The structure of the $3^{\prime}-{ }^{-11} \mathrm{UUGU}^{-8}-5^{\prime}$ template strand promoter motif is well resolved in both cryo-EM and X-ray electron density maps, although ${ }^{-8} \mathrm{U}$ is partially disordered in the cryo-EM map (Fig. 7a, 7b). The most critical and obligatory ${ }^{-10} U$ base, the replacement of which by a $T$ leads to the abolishment of promoter-specific transcription ${ }^{3}$ (Fig. 1c), is buried in a deep pocket at the interface of the gp226 2.1bis helix and $\beta \mathrm{N}$ gp105 (Fig. 2a, 2c, 8a). In this pocket, the ${ }^{-10} \mathrm{U}$ base is wedged between the side chains of gp226 I207 and $\beta N$ gp105 R363 (Fig. 8a), forming a stacking interaction with the latter. Its Watson-Crick interface forms hydrogen bonds with the side chain of $\beta \mathrm{N}$ gp $105 \mathrm{~K} 375$ and with the main chain N of gp226 V206 and I207. Most importantly, the C5 atom of the ${ }^{-10} \mathrm{U}$ pyrimidine ring is only $3.9 \AA$ away from the C $\beta$ of V206, suggesting that a C5 position methyl group would clash with the V206 side chain (Fig. 8a). Accordingly, a holoenzyme containing gp226 with a V206G substitution recognized ${ }^{-10} \mathrm{U}$ - and ${ }^{-10} \mathrm{~T}$-containing promoters with equal efficiencies (Fig. 8b). Notably, all close homologs of gp226 proteins in jumbo phages with deoxyuridine-containing genomic DNA ${ }^{5,6}$ display high sequence conservation of the 2.1 bis helix with the critical valine being absolutely conserved. This suggests that these phages employ a similar mechanism for uracil-dependent promoter recognition. 
The requirement of $\mathrm{U}$ vs. $\mathrm{T}$ in the $-11^{\text {th }}$ position of the promoter is nearly as strong as in the $-10^{\text {th }}$ position. Additionally, a $\mathrm{G}$ is required in the $-9^{\text {th }}$ position ${ }^{3}$. However, the enzyme displays almost no $\mathrm{U}$ vs. $T$ preference in the $-8^{\text {th }}$ position (Fig. 1c). In the promoter complex, the bases of ${ }^{-11} \mathrm{U}$ and ${ }^{-9} \mathrm{G}$ form a stacking interaction such that the $\mathrm{C} 5$ and $\mathrm{C} 6$ atoms of ${ }^{-11} \mathrm{U}$ butt against the sugar-phosphate backbone of the ${ }^{-11} \mathrm{UUG}^{-9}$ segment, leaving no space for a $\mathrm{C} 5$ position methyl group (Fig. 8a). There is a stacking interaction between ${ }^{-9} \mathrm{G}$ and the phenol ring of gp226 Y210 (which belongs to the $2.1 \mathrm{bis}$ helix), and there are three hydrogen bonds between the Watson-Crick interface of ${ }^{-9} \mathrm{G}$ and the main chain of gp226 residues F261 and Y263, which provides a rationale for the G requirement in this position. ${ }^{-8} \mathrm{U}$ forms one hydrogen bond with ${ }^{-11} \mathrm{U}$ and one hydrogen bond with the tip of the $\beta^{\prime}$ lid, which is longer than in its bacterial counterparts, as described above. The $\mathrm{C} 5$ position of ${ }^{-8} \mathrm{U}$ points into solution and can accommodate the additional methyl group of T (Fig. 8a).

\section{Interaction with the non-template strand is essential for the transcription of dsDNA}

The gp226 NTD displays several deep pockets that capture the ss part of the upstream oligonucleotide, which mimics the non-template strand of the transcription bubble (Fig. 8c). In the transcription bubble (Fig. 2b), this part of the non-template strand must have a sequence complementary to the template strand promoter motif $\left(5^{\prime}-{ }^{-11} \mathrm{AACA}^{-8}-3^{\prime}\right.$, the numbering is relative to the TSS). Our oligonucleotide contained a similar motif in its ss part $\left(5^{\prime}-{ }^{-1} \mathrm{AATA}^{-4}-3^{\prime}\right.$, same numbering as the downstream nucleotide, Fig. 2b). Together with its neighboring bases this sequence matched the appearance of the electron density. The base of the mismatched third position nucleotide ( $\mathrm{T} \leftrightarrow \mathrm{C}$ ) does not interact with the gp226 NTD but instead protrudes into solution (Fig. 8c). Despite being only partially complementary to the promoter, this motif was likely a key determinant in the fortuitous binding of the upstream oligonucleotide. 
The gp226 NTD interacts with the backbone and bases of the non-template DNA strand of the transcription bubble via pi-pi stacking, ion pairs, and hydrogen bonds. The length of this interface exceeds $30 \AA$. The extent of these interactions suggests that they play an important role in promoter recognition of a dsDNA template. Indeed, a Y246A substitution, which eliminated pi-pi stacking between the side chain of Y246 and the ${ }^{-2} \mathrm{~A}$ base of the ${ }^{-1} \mathrm{AATA}^{-4}$ motif, abolished transcription on dsDNA but did not affect transcription on a fork template (Fig. 8c, 8d). Furthermore, a S245E substitution introduced a large, negatively charged side chain on the surface of the gp226 NTD that interfered with the trace and conformation of the sugar-phosphate backbone between ${ }^{-2} \mathrm{~A}$ and ${ }^{-4} \mathrm{~A}$. As a consequence, the transcriptional activity of the holoenzyme containing the S245E gp226 mutant on a dsDNA template was weak, whereas its fork template activity was at or above that of WT (Fig. 8d).

In the cryo-EM structure of the DNA template-free AR9 nvRNAP holoenzyme, the NTD and $\sigma$ finger-like element of gp226 are disordered (Fig. 5a, 5b). Similarly to the TL insertion domain, the disorder is likely due to positional heterogeneity since both the gp226 NTD and TL insertion domain possess well-defined hydrophobic cores and are folded in other states of the nvRNAP complex. Furthermore, the NTD is resistant to proteolysis by trypsin in gp226 recombinantly expressed on its own (Fig. 1d). The order-disorder transitions of the gp226 NTD and $\sigma$ finger-like element play a role in the promoter recognition mechanism described below.

\section{Gp226 CTD interacts with dsDNA in a sequence independent manner}

Although weak, the cryo-EM and X-ray electron density of the upstream oligonucleotide stretches from the $\sigma_{2}$-like part of the gp226 NTD to the gp226 CTD (Fig. 7c, 7d). This interaction is about 35 DNA base pairs upstream from the TSS drawing a parallel to the -35 consensus element of bipartite bacterial promoters. The recognition of the -35 element by bacterial $\sigma$ factors is mediated by a helix-turn-helix motif of the $\sigma_{4}$ domain $^{25}$ (Fig. 6b), which interacts with the major groove of dsDNA ${ }^{26}$ 
in a sequence specific manner. AR9 nvRNAP promoters, however, display no sequence conservation in this region (Fig. 1b) and, accordingly, the gp226 CTD interacts with the minor groove of dsDNA which displays few sequence-specific features in the $\mathrm{B} \mathrm{form}{ }^{27}$. Furthermore, this interaction is mediated by a scrunched $\beta$-strand (Fig. 6a) that carries several positively charged residues (R389, K390, R394, K395, K396), but not by a helix-turn-helix motif, which is absent from the gp226 structure. We termed the DNA interacting element of the gp226 CTD (amino acids 386-395) a pseudo- ${ }^{-35}$ element-binding motif.

To examine the role of the pseudo- ${ }^{-35}$ element-binding motif in promoter recognition, we removed most of the positive charge displayed on its surface by replacing R389, K390, R394, K395, and K396 of gp226 with alanines (we called this mutant $A^{5}$ ) (Fig. 8e, 8f). As the AR9 nvRNAP holoenzyme containing $\mathrm{A}^{5}$ gp226 had a lower activity overall, we compared its activity to that of the wild type (WT) holoenzyme on two dsDNA templates that either contained or lacked the upstream part required for interaction with the pseudo- ${ }^{-35}$ element-binding motif (the $[-60,+80]$ and $[-16,+80]$ templates in Fig. 8g, respectively). The WT enzyme exhibited a greater decrease in activity on the $[-16,+80]$ template compared to that of the mutant, which shows that the pseudo- ${ }^{-35}$ element-binding motif is essential for optimal promoter recognition.

The AR9 gp226 pseudo- ${ }^{-35}$ element-binding motif maps onto a disordered part of phage phiKZ gp68 $8^{21}$ (residues 413-429, Supplementary Fig. 2a). This region of gp68's surface carries a positive charge, akin to that of AR9 gp226 (Fig. 8e), even without the inclusion of disordered residues in electrostatic potential calculations. Again, analogous to the AR9 nvRNAP, phiKZ nvRNAP promoters show no sequence conservation 35 nucleotides upstream of the TSS ${ }^{28}$. Considering i) the homology of the phiKZ and AR9 nvRNAPs to cellular RNAPs ${ }^{21}$, ii) the homology of AR9 gp226 to phiKZ gp68 described above, and iii) the presence of positive charges at equivalent locations on the surface of the AR9 gp226 and phiKZ gp68 CTDs, the phiKZ nvRNAP is thus likely to form an AR9 nvRNAP-like 
transcription bubble in which the CTD of gp68 participates in the binding of upstream dsDNA. Furthermore, as all these properties are seemingly conserved for such distantly related viruses as AR9 and phiKZ that infect unrelated hosts (Gram positive B. subtilis and Gram negative Pseudomonas aeruginosa, respectively), and have different genomic DNA base composition ${ }^{1,29}$ and genome replication strategies ${ }^{30}$, the supposition of AR9 nvRNAP-like transcription bubbles can be extended to nvRNAPs of all jumbo phages.

\section{Free energy of template strand promoter binding}

To reconcile the tight integration of AR9 nvRNAP promoter DNA into the promoter complex (Fig. 2a, 2c, 8a) with the transient nature of this complex, we examined the binding free energy of the three best-ordered promoter nucleotides $3^{\prime}-{ }^{-11} U_{U G}{ }^{-9}-5^{\prime}$ to the AR9 nvRNAP holoenzyme by executing

a double decoupling method molecular dynamics protocol ${ }^{31-34}$. The procedure assumes that the conformation of the enzyme does not appreciably change upon promoter binding. As such, the simulations describe a state in which the gp226 NTD has associated with the AR9 nvRNAP core.

The standard binding free energy was calculated by combining the results of four separate simulations that corresponded to the vertical reactions in the thermodynamic cycle shown in Fig. 9a. After the equilibration of the system (Fig. 9b, 9c), two types of simulations were performed: i) "alchemical transformations" in which the occupancy of the oligonucleotide located either in the promoter pocket $\left(\Delta G_{\text {alchemical }}^{\text {bound }}\right)$ or in bulk water $\left(\Delta G_{\text {alchemical }}^{\text {bulk water }}\right)$ was reduced to zero while the oligonucleotide was harmonically constrained to maintain the promoter pocket bound conformation (Fig. 9d, 9e), and ii) calculations of the entropic cost of such harmonic constraints for an oligonucleotide located in the promoter pocket $\left(\Delta G_{\text {restrain }}^{\text {bound }}\right)\left(\right.$ Fig. 9f-9l) and in bulk water $\left(\Delta G_{\text {restrain }}^{\text {bulk }}\right)($ Fig. 9m). To ensure reproducibility and to minimize bias, all simulations were run bidirectionally. 
The favorable energetics of promoter binding via alchemical transformation $(-12.7 \pm 2.3$ kcal/mol, Fig. 9d, 9e) are partially offset by the unfavorable entropic contributions of constraints on DNA conformation and position $(5.8 \pm 1.5 \mathrm{kcal} / \mathrm{mol}$, Fig. 9f-9m). The resulting free energy gain upon complex formation is $-6.9 \pm 2.8 \mathrm{kcal} / \mathrm{mol}$, which shows that the interaction of this promoter element with the enzyme is relatively weak. Thus, despite its unusual structure in which the ${ }^{-10} U$ is buried in a deep pocket and ${ }^{-9} \mathrm{G}$ and ${ }^{-11} \mathrm{U}$ form a stacking interaction, the promoter complex is transient, and the enzyme can easily proceed towards elongation.

\section{The mechanism of template strand promoter recognition in dsDNA}

Combining these findings, we propose the following model for promoter recognition by the AR9 nvRNAP (Fig. 10). In the free state of the AR9 nvRNAP holoenzyme molecule, the NTD of gp226 is folded but does not interact with the body of the enzyme (it is positionally disordered or mobile) and the promoter-binding pocket is absent (Supplementary Fig. 3). The NTD of gp226 is attached to the CTD and the core via a linker that will eventually form a $\sigma$ finger-like structure in the promoter complex. The enzyme displays two positively charged patches that have DNA binding propensity on their surface the pseudo- ${ }^{-35}$ element-binding motif and a patch with a much stronger positive charge and better shape complementarity for the binding of a non-template strand motif that is complementary to the promoter (Supplementary Fig. 3 and State 1 in Fig. 10).

The process of promoter recognition contains the following steps. 1) The gp226 NTD captures the non-template strand in a groove on its surface by recognizing a partially promoter-complementary motif and enabling the initial melting of template dsDNA (State 2 in Fig. 10). Both events are facilitated by the AU-rich promoter-containing regions, which transiently display flipped-out bases. Only three bases of this motif interact with the protein (the third position base ${ }^{-9} \mathrm{C}$ does not, Fig. 8c). The prevalence of the 5'-AANA-3' motif in the AR9 genome (18,972 instances or every 26 nucleotides) may allow the 
enzyme to scan the template. 2) The pseudo- ${ }^{-35}$ element-binding motif of the gp226 CTD interacts with dsDNA, reducing the conformational space available to the gp226 NTD, and thus promoting its binding to the body of the enzyme (State 3 in Fig. 10). 3) The NTD of gp226 comes in contact with the body of the enzyme, fully separating the DNA strands, forming a $\sigma$ finger-like element and a transcription bubble, and placing the template strand at the [gp226]:[ßN gp105] interface. This interface captures a flipped-out ${ }^{-10} \mathrm{U}$ base and buries it into the ${ }^{-10} \mathrm{U}$ recognition pocket. Simultaneously, the DNA strand is squeezed slightly such that the bases flanking the flipped-out ${ }^{-10} U$ base form a stack, and the identities of the stacked ${ }^{-9} \mathrm{G}$ and ${ }^{-11} \mathrm{U}$ bases are verified via geometry-sensitive interactions (hydrogen bonds and ion pairs). Additional interactions are formed at the catalytic center where the TSS is recognized (State 4 in Fig. 10). As the free energy of promoter recognition is nevertheless reasonably low and the conformation of the sugar-phosphate backbone for the four nucleotides of the promoter motif is close to that of dsDNA, the enzyme can efficiently proceed with elongation.

\section{Conclusion}

Here we have explained the functional mechanism of a phage-encoded RNAP that contains a unique promoter-specificity subunit, recognizes the promoter in the template strand of DNA, requires uracil bases in the promoter, and does not use a common helix-turn-helix motif for the binding of dsDNA. Even though the AR9 nvRNAP and its promoter specificity subunit appear to have a common ancestor with their bacterial counterparts, the extent to which the AR9 nvRNAP promoter recognition mechanism is different from that of any known RNAP shows that our knowledge of the structure and function of these complex enzymes is far from complete. 


\section{Methods}

No statistical methods were used to predetermine sample size. The experiments were not randomized and the investigators were not blinded to allocation during experiments and outcome assessment.

\section{Cloning of the AR9 nvRNAP and its mutants}

Four gene-Blocks (gBlocks) encoding AR9 nvRNAP core enzyme genes optimized for expression in E. coli were synthetized by Integrated DNA Technologies (IDT). These gBlocks were assembled into an expression vector on the pETDuet-1 plasmid backbone with the help of the NEBuilder HiFi DNA Assembly Master Mix (New England Biolabs). First, pETDuet-1 was digested by the NcoI and BamHI endonucleases, and gBlocks coding for N-terminally hexahistidine-tagged gp270 and gp154 were ligated. Then, this plasmid was digested by the BglII and XhoI endonucleases and ligated with two gBlocks coding for gp105 and gp089. The resulting plasmid encoded the AR9 nvRNAP core enzyme.

The plasmid for expression of the AR9 nvRNAP holoenzyme was created by inserting an E. colioptimized gp226 gBlock (also synthetized by ITD) into the AR9 nvRNAP core plasmid described above, which was linearized at the XhoI site. This plasmid was used as a template to create mutant versions of the AR9 nvRNAP holoenzyme by site-directed mutagenesis (the list of corresponding primers is in the

\section{Supplementary Table 3).}

The plasmid encoding the tagless AR9 nvRNAP core enzyme was derived from the His-tagged AR9 nvRNAP core plasmid described above. First, a fragment that contained all the four genes but excluded the Hig-tag was PCR amplified (the primers are listed in the Supplementary Table 3). Then, the pETDuet-1 vector was linearized by the NcoI and XhoI endonucleases. A new plasmid was then 
created by ligating the PCR fragment and the linearized pETDuet-1 vector using the NEBuilder HiFi DNA Assembly Master Mix (New England Biolabs).

In all plasmids, a T7 RNAP promoter, a lac operator, and a ribosome binding site were located at appropriate positions upstream of each gene.

\section{Purification of recombinant AR9 nvRNAP}

Plasmids encoding AR9 nvRNAP core, tagless AR9 nvRNAP core, and holoenzyme or its mutants were transformed into BL21 Star (DE3) chemically competent $E$. coli cells. The cultures (3 L) were grown at $37^{\circ} \mathrm{C}$ to $\mathrm{OD}_{600}$ of 0.7 in $\mathrm{LB}$ medium supplemented with ampicillin at a concentration of $100 \mu \mathrm{g} / \mathrm{mL}$, and recombinant protein overexpression was induced with $1 \mathrm{mM}$ IPTG for 4 hours.

Cells containing over-expressed AR9 nvRNAP holoenzyme or its mutants were harvested by centrifugation and disrupted by sonication in buffer A (40 mM Tris- $\mathrm{HCl} \mathrm{pH} 8,300 \mathrm{mM} \mathrm{NaCl}, 3 \mathrm{mM} \beta$ mercaptoethanol) followed by centrifugation at 15,000 g for $30 \mathrm{~min}$. Cleared lysate was loaded onto a 5 mL HisTrap sepharose HP column (GE Healthcare) equilibrated with buffer A. The column was washed with buffer A supplemented with $20 \mathrm{mM}$ Imidazole. The protein was eluted with a linear 0-0.5 M Imidazole gradient in buffer A. Fractions containing AR9 nvRNAP holoenzyme or its mutants were combined and diluted with buffer B (40 mM Tris-HCl pH 8, 0.5 mM EDTA, 1 mM DTT, 5\% glycerol) to the $50 \mathrm{mM} \mathrm{NaCl}$ final concentration and loaded on equilibrated $5 \mathrm{~mL}$ HiTrap Heparin HP sepharose column (GE Healthcare). The protein was eluted with a linear 0-1 M NaCl gradient in buffer B. Fractions containing AR9 nvRNAP holoenzyme or its mutants were pooled and concentrated (Amicon Ultra-4 Centrifugal Filter Unit with Ultracel-50 membrane, EMD Millipore) to a final concentration of $3 \mathrm{mg} / \mathrm{mL}$, then glycerol was added up to $50 \%$ to the sample for storage at $-20^{\circ} \mathrm{C}$ (the samples were used for transcription assays). 
Samples used for crystallization and cryo-EM were produced by following a slightly different procedure. Cells containing over-expressed recombinant AR9 nvRNAP core or holoenzyme were harvested by centrifugation and disrupted by sonication in buffer $\mathrm{C}\left(50 \mathrm{mM} \mathrm{NaH}_{2} \mathrm{PO}_{4} \mathrm{pH} 8,300 \mathrm{mM}\right.$ $\mathrm{NaCl}, 3 \mathrm{mM} \beta$-mercaptoethanol, $0.1 \mathrm{mM}$ PMSF) followed by centrifugation at $15,000 \mathrm{~g}$ for $30 \mathrm{~min}$. Cleared lysate was loaded on $5 \mathrm{~mL}$ Ni-NTA column (Qiagen) equilibrated with buffer C, washed with 5 column volumes of buffer $\mathrm{C}$ and with 5 column volumes of buffer $\mathrm{C}$ containing $20 \mathrm{mM}$ Imidazole. Then, elution with buffer C containing $200 \mathrm{mM}$ Imidazole was carried out. Fractions containing AR9 nvRNAP core or holoenzyme were pooled and diluted ten times by buffer D (20 mM Tris $\mathrm{pH} 8,0.5 \mathrm{mM}$ EDTA, $1 \mathrm{mM}$ DTT) or by buffer E (20 mM Bis-tris propane $\mathrm{pH}$ 6.8, $0.5 \mathrm{mM}$ EDTA, $1 \mathrm{mM}$ DTT) correspondingly and applied to a MonoQ 10/100 column (GE Healthcare). Bound proteins were eluted with a linear $0.25-0.45 \mathrm{M} \mathrm{NaCl}$ gradient in buffer $\mathrm{D}$ or E correspondingly.

Cells containing over-expressed recombinant tagless AR9 nvRNAP core were harvested by centrifugation and disrupted by sonication in buffer B followed by centrifugation at $15,000 \mathrm{~g}$ for $30 \mathrm{~min}$. An 8\% polyethyleneimine (PEI) solution ( $\mathrm{pH} 8.0$ ) was added with stirring to the cleared lysate to the final concentration of $0.8 \%$. The resulting suspension was incubated on ice for 1 hour and centrifuged at $10,000 \mathrm{~g}$ for $15 \mathrm{~min}$. The supernatant was removed and the pellet was resuspended in buffer B containing $0.3 \mathrm{M} \mathrm{NaCl}$. After 10 min incubation, the PEI pellet was formed by centrifugation as previously. Then, supernatant was removed and the pellet was resuspended in buffer B containing $1 \mathrm{M}$ $\mathrm{NaCl}$ followed by centrifugation at $10,000 \mathrm{~g}$ for $15 \mathrm{~min}$. Eluted proteins were precipitated the supernatant by addition of ammonium sulfate to $67 \%$ saturation and dissolved in buffer $\mathrm{D}$ and loaded on equilibrated 5 mL HiTrap Heparin HP sepharose column (GE Healthcare). The protein was eluted with a linear 0-1 $\mathrm{M} \mathrm{NaCl}$ gradient in buffer $\mathrm{D}$. Fractions containing tagless AR9 nvRNAP core were pooled and subjected to anion exchange chromatography as described above for AR9 nvRNAP core. 
The AR9 nvRNAP core sample was polished and buffer-exchanged using size exclusion chromatography on a Superdex 200 10/300 (GE Healthcare) column equilibrated with buffer D containing $100 \mathrm{mM} \mathrm{NaCl}$. The tagless AR9 nvRNAP core and AR9 nvRNAP holoenzyme were not subjected to size exclusion chromatography - salt concentration in the sample was lowered during the concentration procedure.

The fractions containing AR9 nvRNAP core, tagless AR9 nvRNAP core or holoenzyme were concentrated to a final concentration of $20 \mathrm{mg} / \mathrm{mL}$ and used for crystallization or cryo-EM.

\section{Preparation of the promoter complex for structure determination}

A forked DNA template for crystallization and cryo-EM work was obtained by hybridization of two linear oligonucleotides given in Supplementary Table 3. The 18 base-long ss part of this forked template contained the AR9 late promoter P077, while its 14 bp-long ds segment spanned positions from +3 to +16 relative to the TSS (Fig. 8b). Both oligonucleotides were synthetized by IDT with dual PAGE and HPLC purification at a final concentrations of $100 \mu \mathrm{M}$ each. They were annealed together in a buffer containing $20 \mathrm{mM}$ Bis-tris propane $\mathrm{pH} 6.8,100 \mathrm{mM} \mathrm{NaCl}, 4 \mathrm{mM} \mathrm{MgCl} 2,0.5 \mathrm{mM}$ EDTA, which was incubated at $65{ }^{\circ} \mathrm{C}$ for 1 minute and then cooled down to $4{ }^{\circ} \mathrm{C}$ at a rate of $1{ }^{\circ} \mathrm{C}$ per minute. A 1.5 -fold molar excess of the DNA template was added to the holoenzyme and incubated for $30 \mathrm{~min}$ at room temperature. The final concentrations of the reagents were as follows: $10 \mathrm{mg} / \mathrm{mL}$ for the protein $(34 \mu \mathrm{M})$ and $50 \mu \mathrm{M}$ for the DNA template. This specimen was used for crystallization and cryo-EM work directly.

\section{Crystallization of AR9 nvRNAP}

The initial crystallization screening was carried out by the sitting drop method in 96 well ARI Intelliwell-2 LR plates using Jena Bioscience crystallization screens at $19^{\circ} \mathrm{C}$. PHOENIX pipetting robot (Art Robbins Instruments, USA) was employed for preparing crystallization plates and setting up drops, 
each containing $200 \mathrm{~nL}$ of the protein and the same volume of well solution. Optimization of crystallization conditions was performed in 24 well VDX plates and thin siliconized cover slides (both from Hampton Research) by hanging drop vapor diffusion. The best crystals were obtained as follows: i) an $1.5 \mu 1$ aliquot of AR9 nvRNAP core $(4.5 \mathrm{mg} / \mathrm{mL})$ was mixed with an equal volume of a solution containing $100 \mathrm{mM}$ Tricine $\mathrm{pH} 8.8,270 \mathrm{mM} \mathrm{KNO}_{3}, 15 \%$ PEG 6000, $5 \mathrm{mM} \mathrm{MgCl}_{2}$, and incubated as a hanging drop over the same solution; ii) an $1.5 \mu 1$ aliquot of tagless AR9 nvRNAP core $(7.5 \mathrm{mg} / \mathrm{mL})$ was mixed with an equal same volume of a solution containing $150 \mathrm{mM}$ Malic acid $\mathrm{pH} 7,150 \mathrm{mM} \mathrm{NaCl}$, $14 \%$ PEG 3350 and incubated as a hanging drop over the same solution; iii) an $1.5 \mu 1$ aliquot of the AR9 nvRNAP promoter complex $(10 \mathrm{mg} / \mathrm{mL})$ was mixed with an equal same volume of a solution containing $150 \mathrm{mM}$ MIB pH 5, $150 \mathrm{mM} \mathrm{LiCl}, 13 \%$ PEG 1500 and incubated as a hanging drop over the same solution. Some crystal reached their final size the next day and some grew for two weeks at 19 $\mathrm{C}^{\circ}$ temperature.

\section{Preparation of heavy-atom derivative crystals}

The following compounds were tested for heavy atom derivatization of AR9 nvRNAP core crystals (by co-crystallization and soaking): $\mathrm{SrCl}_{2}, \mathrm{GdCl}_{3}, \mathrm{Na}_{2} \mathrm{WO}_{4}, \mathrm{HgCl}_{2}, \mathrm{~Pb}\left(\mathrm{NO}_{3}\right)_{2}$, thimerosal (2$\left.\left(\mathrm{C}_{2} \mathrm{H}_{5} \mathrm{HgS}\right) \mathrm{C}_{6} \mathrm{H}_{4} \mathrm{CO}_{2} \mathrm{Na}\right), 10$ compounds containing Eu and $\mathrm{Yb}$ atoms (JBS Lanthanide Phasing Kit), three compounds containing W (JBS Tungstate Cluster Kit) and one cluster compound containing Ta $\left(\mathrm{Ta}_{6} \mathrm{Br}_{12}\right.$ JBS Tantalum Cluster Derivatization Kit). The crystals were soaked in a range of concentrations of heavy atom compounds (between $0.1 \mathrm{mM}$ and $100 \mathrm{mM}$ ) that were added to the crystallization solution. The soaking time was varied from 2 hours to 2 days. Among all examined conditions, only solutions containing $10 \mathrm{mM}$ thimerosal or $1 \mathrm{mM}$ tantalum bromide resulted in heavy atom derivatization (judging by the presence of anomalous signal in X-ray diffraction data) upon overnight soaking. 
To produce a Se-methionine (SeMet) derivative of the AR9 nvRNAP core enzyme, the corresponding plasmid was transformed into B834(DE3) chemically competent E. coli cells. The cells were first grown in LB medium until the optical density $\mathrm{OD}_{600}$ reached a value of 0.35 . The cells were then pelleted by centrifugation at $4,000 \mathrm{~g}$ for $10 \mathrm{~min}$ at $4^{\circ} \mathrm{C}$ and transferred to the SelenoMet Medium (Molecular Dimensions) that was supplemented with ampicillin at a concentration of $100 \mu \mathrm{g} / \mathrm{mL}$. The protein expression then proceeded according to the manufacturer's instructions. All the subsequent steps were the same as for the native protein.

\section{X-ray data collection}

Cryoprotectant solutions were prepared by replacing $25 \%$ of water in the crystallization solution (hanging drop well solution) with ethylene glycol, which was found to be the best cryoprotectant by trial and error. The crystals were either soaked for 1-5 minutes in the cryoprotectant solution or briefly dipped into it and then flash frozen in liquid nitrogen. Such frozen crystals were then transferred to a shipping Dewar and shipped to the APS (LS-CAT) or ALS (BCSB) synchrotrons for remote data collection. Xray diffraction data and fluorescent spectra were collected in a nitrogen stream at $100 \mathrm{~K}$.

Heavy atom and SeMet derivative data were collected at the absorption peak wavelength (the white line, if present) of the X-ray fluorescence spectrum.

\section{$\mathrm{X}$-ray structure determination}

The structure determination process spanned nearly three years. Initially, we aimed to solve the structure of the AR9 nvRNAP core enzyme by X-ray crystallography or cryo-EM and use it to solve the structure of the promoter complex. However, the atomic model of the promoter complex obtained by cryo-EM was built first. Then, it was used to solve the X-ray structure of the core and to interpret the cryo-EM map of the holoenzyme. The path to the atomic model described below is the trunk of a tree 
that had many branches representing things that did not work. The following procedure involves fewest datasets and fewest steps that lead to an interpretable map.

None of the RNAP structures present in the PDB at the start of this project were sufficiently similar to solve the structure of the AR9 nvRNAP core by molecular replacement (MR), so crystallographic phases had to be obtained by a de novo phasing procedure (heavy atom isomorphous replacement or anomalous scattering). Severe anisotropy and inconsistent diffraction of AR9 nvRNAP core native crystals made this task extremely complicated and we had to screen hundreds of heavy atomsoaked crystals for diffraction. The SeMet derivative diffracted to $5.5 \AA$ resolution, which was insufficient to solve the Se substructure using anomalous scattering. Moreover, this derivative was not isomorphous to any of the native datasets.

An interpretable map was obtained by a convoluted procedure. A map in which the characteristic features of a DNA-dependent RNAP - two adjoining double- $\psi \beta$-barrel (DPBB) domains and several large $\alpha$-helices, including the bridge helix (although split in the middle) - could be discerned, but no side chain densities were present, was obtained by a multiple isomorphous replacement plus anomalous scattering method which was applied to the native, $\mathrm{Ta}_{6} \mathrm{Br}_{12}$, and thimerosal derivative datasets of the His-tagged AR9 nvRNAP core enzyme (see Supplementary Table 1). This map was calculated by the SHARP software package that was run with mostly default settings ${ }^{35}$. The most similar part ${ }^{36}$ of the archaeal RNAP structure ${ }^{37}$ (PDB code 4ayb) was fitted into this density using $\operatorname{Coot}^{38}$ and all parts that did not fit the density and all side chains were removed. The resulting model contained 832 alanine residues.

This model was then used to solve the structure of a unique thimerosal derivative dataset that belonged to a different unit cell (Supplementary Table 1) with the help of a MR procedure ${ }^{39}$. This unique dataset resulted from the crystallization of a tagless version of the AR9 nvRNAP core (all native 
$\mathrm{N}$ - and C-termini). It had a very large orthorhombic unit cell with eight molecules of the AR9 nvRNAP core in its asymmetric unit or about 17,800 amino acids. Remarkably, Phaser $^{40}$ was able to locate all eight copies of the AR9 nvRNAP core in this $3.8 \AA$ resolution dataset with help of the 832-residue polyalanine fragment (obtained as described before) as a search model. This polyalanine search model corresponded to about $1.8 \%$ of the total protein material in the asymmetric unit and $1 \%$ of the total asymmetric unit content if solvent atoms are taken into account. The density was then dramatically improved by 25 cycles of eightfold non-crystallographic symmetry (NCS) averaging using Parrot ${ }^{41}$. The resulting density was mostly continuous and showed many bulky side chains especially in the vicinity of the DPBB domains. Buccaneer ${ }^{42}$ was then used for automatic model building into this map. The Buccaneer model was cleaned up manually and separate chain fragments were assembled into a new intermediate AR9 nvRNAP core model that contained 995 residues of which 937 had side chains. The new intermediate model was then used as a search model in a new round of MR by Phaser that was followed by NCS averaging using Parrot.

The new density was of sufficient quality to recognize the identity of many side chains and for manual model building using Coot. Structures of the Mycobacterium tuberculosis and E. coli RNAPs (PDB codes $5 \mathrm{ZX}^{43}$ and $6 \mathrm{C}^{4} \mathrm{Y}^{44}$, respectively) were used to aid in chain tracing. Additionally, this thimerosal derivative dataset contained $\mathrm{Hg}$ atoms, identified with the help of anomalous Fourier synthesis, that were expected to bind to cysteine side chains. Thus, the $\mathrm{Hg}$ atoms were used as markers to maintain the chain register.

Eventually, a large fraction of the nvRNAP core atomic model was complete. Some peripheral parts, however, and the $\beta^{\prime} \mathrm{C}$ gp154 Insertion domain were too disordered for model building. While this work was in progress, an interpretable (3.8 $\AA$ resolution) cryo-EM map of the AR9 nvRNAP promoter complex was obtained. This map was of better quality than the $3.8 \AA$ resolution, large unit cell, X-ray 
dataset of the tagless core enzyme, so further rounds of model building were continued using the cryoEM map. Once the holoenzyme model was complete, the core or the entire holoenzyme (but not the DNA) were used to solve the crystal structure of the native AR9 nvRNAP core (3.3 $\AA$ resolution, the standard unit cell, Supplementary Table 1) and promoter complex (3.4 Å resolution, Supplementary Table 1) by MR using Phaser.

Some peripheral regions of the cryo-EM promoter complex map, namely, the $\beta^{\prime} \mathrm{C}$ gp 154 Insertion domain, residues 130-289 of $\beta \mathrm{N}$ gp105, and the peripheral parts of gp226, were too poor for reliable de novo model building. Fortunately, the structure of AR9 nvRNAP promoter complex was one of large multisubunit targets of the CASP14 protein structure prediction competition. The Google DeepMind AlphaFold2 software predicted the structure of difficult-to-build domains with excellent accuracy ${ }^{23}$. This allowed us to complete the AR9 nvRNAP promoter complex model in the cryoEM map first and then use this model to solve the $3.4 \AA$ resolution crystal structure of the promoter complex.

Refinement of crystallographic and cryo-EM models was performed using Phenix ${ }^{45}$ and Coot. Additional details describing model building and the analysis of AlphaFold2 models are given elsewhere ${ }^{46}$.

\section{Cryo-EM sample preparation and data acquisition of the AR9 nvRNAP promoter complex}

QUANTIFOIL 1.2/1.3 copper grids were plasma cleaned for 30s using the model 950 advanced plasma system by Gatan. $3 \mu \mathrm{L}$ of $10 \mathrm{mg} / \mathrm{mL}$ of the AR9 nvRNAP holoenzyme $(34 \mu \mathrm{M})$ and $50 \mu \mathrm{M}$ of the DNA nucleotide in $20 \mathrm{mM}$ Bis-tris propane $\mathrm{pH} 6.8,100 \mathrm{mM} \mathrm{NaCl}, 2 \mathrm{mM} \mathrm{MgCl}, 0.5 \mathrm{mM}$ EDTA was pipetted onto to the grid and blotted using a Vitrobot (Thermo Fischer Scientific) at 100\% humidity for $5 \mathrm{~s}$. Following blotting, the sample was plunged into liquid ethane cooled by liquid nitrogen. 
5,351 (5,760 × 4,092 pixels) micrograph movies were collected using the EPU software on a Titan Krios $300 \mathrm{kV}$ electron microscope with a BioQuantum K3 imaging filter with a $20-\mathrm{eV}$ slit. Each movie contained 56 frames collected over $1.5 \mathrm{~s}$, with a frame dose of $0.78 \mathrm{e} / \AA^{2}$ and pixel size of $1.09 \AA$. Movies were collected over a defocus range of -1 to $-4 \mu \mathrm{m}$ (Supplementary Table 2).

\section{Cryo-EM image processing of the AR9 nvRNAP promoter complex}

Image processing was performed using Eman2 ${ }^{47}$, Relion3.0 ${ }^{48}$ and CryoSPARC3.0 ${ }^{49}$ (Supplementary Fig. 4, Supplementary Table 2). All movies were motion corrected using MotionCor $2{ }^{50}$. Estimation of the contrast transfer function (CTF) parameters was performed by CTFFIND4.1 ${ }^{51}$ over the resolution range of 5.0-30.0 $\AA$. E2boxer ${ }^{47}$ was used for particle picking, resulting in 420,791 particles with a box size of 300 pixels. Particle box coordinates were used by Relion3.0 to extract the boxes. 2D classification by Relion3.0 resulted in 243,976 particles belonging to high quality classes. These particles were imported into CryoSPARC3.0, where ab initio reconstruction was carried out using three models. Following the ab initio reconstruction, 3D classification was performed with five classes, a box size of 150 pixels to improve speed, a batch size of 2,000 particles per class and an assignment convergence criterion of $2 \%$. Non-Uniform (NU) refinement ${ }^{52}$ was executed using both the 106,867 particles and the map from the most populous class of 3D classification. 3D local refinement was then carried out using an alignment resolution of $0.25^{\circ}$ and $\mathrm{NU}$ refinement. Subsequently, local CTF-refinement was performed using a search range of 3.5-20.0 A. Following this, around round of NU refinement and 3D local refinement with an alignment resolution of $0.25^{\circ}$ and NUrefinement was performed. The resulting map was sharpened with a B-factor of $-138 \AA^{2}$.

\section{Cryo-EM sample preparation and data acquisition of the AR9 nvRNAP holoenzyme}

TED PELLA 200 mesh PELCO NetMesh copper grids were plasma cleaned for 30s using the model 950 advanced plasma system by Gatan. $3 \mu \mathrm{l}$ of $20 \mathrm{mg} / \mathrm{ml}$ His-tag $5 \mathrm{~s}$ nvRNAP in $20 \mathrm{mM}$ Bis-tris 
propane $\mathrm{pH} 6.8,100 \mathrm{mM} \mathrm{NaCl}, 4 \mathrm{mM} \mathrm{MgCl} 2,0.5 \mathrm{mM}$ EDTA buffer was pipetted onto to the grid and blotted using a Vitrobot (Thermo Fischer Scientific) at 100\% humidity for $5 \mathrm{~s}$. Following blotting, the sample was plunged into liquid ethane cooled by liquid nitrogen.

2,691 $(3,838 \times 3,710$ pixels $)$ micrograph movies were collected using the EPU software on a Titan Krios 300kV electron microscope with a K2 Summit camera and a $20-\mathrm{eV}$ slit. Each movie contained 40 frames collected over $8 \mathrm{~s}$, with a frame dose of $1.08 \mathrm{e} / \AA^{2}$ and pixel size of $1.08 \AA$ (Supplementary Table 2). Movies were collected with a target defocus of $-1.8 \mu \mathrm{m}$. Images were collected with a 30-degree tilt.

\section{Cryo-EM image processing of the AR9 nvRNAP holoenzyme}

Image processing was performed using Relion $2.0^{48}$. All movies were motion corrected using MotionCor2 ${ }^{50}$. Estimation of the contrast transfer function (CTF) parameters was performed by $\mathrm{Gctf}^{53}$. Particle picking resulted in 227,577 particles with a box size of 200 pixels. 2D and 3D classification by Relion2.0 resulted in 104,471 particles belonging to high quality classes. The resulting particles were refined to a resolution of $4.4 \AA$ (Supplementary Table 2). The resulting map was sharpened with a Bfactor of $-87 \AA^{2}$.

\section{Gp226 cloning, purification and limited digestion with trypsin}

The AR9 gene 226 was PCR amplified from AR9 genomic DNA and cloned into the pQE-2 vector (QIAGEN) between the SacI and SalI restriction sites. The resulting plasmid was transformed into BL21 (DE3) chemically competent $E$. coli cells. The culture $(7 \mathrm{~L})$ was grown at $37^{\circ} \mathrm{C}$ to an $\mathrm{OD}_{600}$ of 0.5 in LB medium supplemented with ampicillin at a concentration of $100 \mu \mathrm{g} / \mathrm{mL}$, and recombinant protein overexpression was induced with $1 \mathrm{mM} \mathrm{IPTG}$ for 4 hours at $22^{\circ} \mathrm{C}$. Cells containing overexpressed recombinant protein were harvested by centrifugation and disrupted by sonication in buffer $\mathrm{C}$ 
followed by centrifugation at $15,000 \mathrm{~g}$ for $30 \mathrm{~min}$. Cleared lysate was loaded on a $5 \mathrm{~mL}$ Ni-NTA column (Qiagen) equilibrated with buffer $\mathrm{C}$, washed with 5 column volumes of buffer $\mathrm{C}$ and with 5 column volumes of buffer $C$ containing $20 \mathrm{mM}$ Imidazole. Then, elution with buffer $\mathrm{C}$ containing $200 \mathrm{mM}$ Imidazole was carried out. Fractions containing gp226 were pooled, concentrated and subjected to gelfiltration on a Superdex 200 10/300 (GE Healthcare) column equilibrated with buffer C. The fractions containing gp226 monomer were concentrated to a final concentration of $1 \mathrm{mg} / \mathrm{mL}$ and used for the limited proteolysis experiment.

Trypsin digestion of gp226, which had a concentration of $80 \mathrm{ng} / \mu \mathrm{L}$, was carried out in $20 \mathrm{ul}$ of the digestion buffer $\left(50 \mathrm{mM} \mathrm{NaH} 2 \mathrm{PO}_{4} \mathrm{pH} 8.0,300 \mathrm{mM} \mathrm{NaCl}\right)$ that contained a range of trypsin concentrations (Sigma-Aldrich). The trypsin to gp226 molar ratios were from 0.03 to 0.6 . The reactions were allowed to proceed for 1 hour at $25^{\circ} \mathrm{C}$ and stopped by the addition of Laemmli loading buffer and immediate boiling. The reaction products were analyzed by denaturing SDS polyacrylamide gel electrophoresis (SDS-PAGE) with subsequent mass-spectrometry as described previously ${ }^{1}$.

\section{DNA templates for transcription assay}

Long DNA templates containing late AR9 promoters were prepared by polymerase chain reaction (PCR). PCRs were done with Encyclo DNA polymerase (Evrogen, Moscow) and the AR9 genomic DNA as a template, with a standard concentration of dNTPs (Thermo Fisher Scientific) to obtain DNA fragments with thymine or in the presence of dUTP (Thermo Fisher Scientific) in place of dTTP to obtain DNA fragments with uracil. Oligonucleotide primers used for PCR are listed in (Supplementary Table 3).

Short double-stranded and partially single-stranded DNA templates containing the P077 promoter with uracils and thymines at certain positions were prepared by annealing of oligonucleotides ordered from Evrogen (Moscow) and listed in (Supplementary Table 3). To prepare specific DNA 
templates, two corresponding oligonucleotides were annealed together by mixing in buffer containing $40 \mathrm{mM}$ Tris- $\mathrm{HCl} \mathrm{pH} 8,10 \mathrm{mM} \mathrm{MgCl}_{2}$ and $0.5 \mathrm{mM} \mathrm{DTT}$, incubating at $75^{\circ} \mathrm{C}$ for 1 minute and cooling down to $4{ }^{\circ} \mathrm{C}$ by a decrement of $1{ }^{\circ} \mathrm{C}$ per minute.

\section{In vitro transcription}

Multiple-round run-off transcription reactions were performed in $5 \mu \mathrm{L}$ of transcription buffer (40 $\mathrm{mM}$ Tris- $\mathrm{HCl} \mathrm{pH}$ 8, $10 \mathrm{mM} \mathrm{MgCl} 2,0.5 \mathrm{mM}$ DTT, $100 \mu \mathrm{g} / \mathrm{mL}$ bovine serum albumin (Thermo Fisher Scientific), and $1 \mathrm{U} / \mu \mathrm{L}$ RiboLock RNase Inhibitor (Thermo Fisher Scientific)) and contained $100 \mathrm{nM}$ AR9 nvRNAP holoenzyme and $100 \mathrm{nM}$ DNA template. The reactions were incubated for $10 \mathrm{~min}$ at $37^{\circ} \mathrm{C}$, followed by the addition of $100 \mu \mathrm{M}$ each of ATP, CTP, and GTP, $10 \mu \mathrm{M}$ UTP and $3 \mu \mathrm{Ci}[\alpha-$ $\left.{ }^{32} \mathrm{P}\right]$ UTP (3000 Ci/mmol) (Fig. 1c, Fig. 8b) or $100 \mu \mathrm{M}$ each of ATP, UTP, GTP, $10 \mu \mathrm{M} \mathrm{CTP}$ and $3 \mu \mathrm{Ci}$ $\left[\alpha-{ }^{32} \mathrm{P}\right] \mathrm{CTP}(3000 \mathrm{Ci} / \mathrm{mmol})$ (Fig. 8d, 8f). Reactions proceeded for $30 \mathrm{~min}$ at $37^{\circ} \mathrm{C}$ and were terminated by the addition of an equal volume of denaturing loading buffer ( $95 \%$ formamide, $18 \mathrm{mM}$ EDTA, $0.25 \%$ SDS, $0.025 \%$ xylene cyanol, $0.025 \%$ bromophenol blue). The reaction products were resolved by electrophoresis on $6-23 \%(\mathrm{w} / \mathrm{v})$ polyacrylamide gel containing $8 \mathrm{M}$ urea. The results were visualized with a Typhoon FLA 9500 scanner (GE Healthcare).

\section{Molecular dynamics general methods}

Simulations were carried out on both the LS5 and Stampede2 systems at the Texas Advanced Computing Center (TACC) using NAMD $2.10^{34}$. The CHARMM36 force field was used ${ }^{54}$. Production runs were performed in the isothermal isobaric (NPT) ensemble using Langevin dynamics and a Langevin piston $^{55}$. Alchemical transformations were analyzed by the ParseFEP package ${ }^{56}$. Entropic restraints were calculated via thermodynamic integration. Collective variables were implemented via the colvars module in $\mathrm{NAMD}^{57}$. The energy of non-bonded VdW interactions for distances exceeding $10 \AA$ was smoothly decreased to equal zero at $12 \AA$. A 2 fs timestep was used in all simulations. Long range 
electrostatics was calculated with the help of the Particle Mesh Ewald algorithm ${ }^{58}$. During alchemical transformation, a soft core VdW radius of $4 \AA^{2}$ was used to improve convergence and accuracy ${ }^{59,60}$. Both alchemical and restraint calculation simulations were carried out bidirectionally. The Bennett Acceptance Ratio maximum likelihood estimate ${ }^{61}$ was used to determine free energy change for alchemical transformations. The double decoupling method (DDM) was implemented as described ${ }^{32,33}$.

\section{Molecular dynamics system setup}

The protein structure description files for both structures - the AR9 nvRNAP holoenzyme in complex with the $3^{\prime}-{ }^{-11} \mathrm{UUG}^{-9}-5^{\prime}$ oligonucleotide bound to the promoter binding pocket and for the $3^{\prime}-$ ${ }^{-11} \mathrm{UUG}^{-9}-5^{\prime}$ oligonucleotide in the promoter bound conformation - were generated using the psfgen plugin of VMD ${ }^{62}$. Solvation was performed using TIP3 water ${ }^{63}$ in a box with $15 \AA$ padding in each direction and ionized in $0.1 \mathrm{M} \mathrm{NaCl}$. The holoenzyme-DNA and DNA systems were enclosed in periodic boxes with cell dimensions of $(176 \AA, 147 \AA, 133 \AA)$ and ( $42 \AA, 42 \AA, 41 \AA)$, respectively, and contained 91,177 and 2,166 water molecules, respectively. Both systems were first minimized for 1,000,000 steps while restraints and constraints on the protein (in the holoenzyme system), DNA, and water atoms were gradually removed. Both systems were heated from $0 \mathrm{~K}$ to $300 \mathrm{~K}$ in $5 \mathrm{~K}$ increments for a total of 19.2 ns with constraints on backbone atoms. The holoenzyme-DNA and DNA systems were then equilibrated with minimal constraints in the isothermal-isobaric (NPT) ensemble for $100 \mathrm{~ns}$ and $50 \mathrm{~ns}$, respectively.

\section{Definition of collective variables}

For the implementation of the DDM method via alchemical transformations, the system must be (harmonically) constrained such that the finite sampling can be focused on relevant regions of phase space. The entropic cost of applying these restraints is evaluated in separate independent simulations. The phase space and the entropic cost are connected to each other through a set of collective variables that are applied to atoms during simulations. 
Only one collective variable is needed to restrain the conformation of the oligonucleotide in bulk water (the bulk water DNA system): the RMSD of all non-H DNA atoms relative to the equilibrated state. In the holoenzyme-DNA complex, seven collective variables are required - six to define the orientation and position of the rigid DNA molecule relative to the holoenzyme complex and one to define the conformation of the DNA. Similarly to the bulk water DNA case, the RMSD of all non-H DNA atoms relative to the equilibrated state is used as the collective variable to restrain the conformation of DNA. We characterize the orientation of the rigid DNA molecule via the relative position of the backbone atoms of ${ }^{-11} \mathrm{UUG}^{-9}$ to those of gp226 V206, gp105 N382 and gp226 K262. Accordingly, the orientation of DNA relative to the holoenzyme is characterized by six collective variables: $r$ - the distance between ${ }^{-11} \mathrm{U}$ and gp226 V206); $\phi$ - the angle between gp226 V206, ${ }^{-11} \mathrm{U}$, and $\left.{ }^{-9} \mathrm{G}\right) ; \chi$ - the angle between ${ }^{-11} \mathrm{U}$, gp226 V206, and gp105 N382); $\theta$ - the dihedral angle between gp226 V206, ${ }^{-11} \mathrm{U},{ }^{-9} \mathrm{G}$, and $\left.{ }^{-10} \mathrm{U}\right) ; \psi-$ the dihedral angle between gp105 N382, gp226 V206, ${ }^{-11} \mathrm{U}$, and $\left.{ }^{-9} \mathrm{G}\right) ; \xi$ - the dihedral angle between ${ }^{-11} \mathrm{U}$, gp226 V206, gp105 N382, and gp226 K262).

The harmonic force constraint constants applied to the distance-type (RMSD and $r$ ) and angular collective variables were $10 \mathrm{kcal} / \mathrm{mol} / \AA^{2}$ and $0.1 \mathrm{kcal} / \mathrm{mol} / \mathrm{deg}^{2}$, respectively. The equilibrium positions for all harmonic restraints were derived from the equilibrated holoenzyme-DNA structure. For restraint estimation simulations, harmonic forces were varied smoothly using a target force exponent value of 4.0. The lambda schedule focused near the value 1.0 to improve simulation convergence and ensure thermodynamic micro-reversibility ${ }^{64,65}:[1.00,0.999,0.99,0.95,0.90,0.85,0.80,0.75,0.70,0.65,0.60$, $0.55,0.50,0.45,0.40,0.35,0.30,0.20,0.10,0.00]$. The reverse sequence was used for the backward simulation.

\section{Thermodynamic cycle}


The standard binding free energy was calculated by combining the results of four separate simulations which represent the four vertical reactions of the thermodynamic cycle (Fig. 9a). These simulations evaluate the following parameters (Supplementary Table 4): 1) the entropic cost of restraining the promoter DNA to the "bound" state in the promoter binding pocket by adding/removing conformational restraints on the promoter DNA $\left.\left(\Delta G_{\text {restrain }}^{\text {bound }}\right) ; 2\right)$ the free energy of coupling/decoupling the promoter DNA from the binding pocket via alchemical transformations with restraints on the conformation of the promoter DNA $\left.\left(\Delta G_{\text {alchemical }}^{\text {bound }}\right) ; 3\right)$ the entropic cost of restraining the promoter DNA to the bound conformation in bulk water by adding/removing conformational restraints on the promoter DNA $\left.\left(\Delta G_{\text {restrain }}^{\text {bulk water }}\right) ; 4\right)$ the free energy of coupling/decoupling the promoter DNA from bulk water via alchemical transformations with restraints on the conformation of the promoter DNA ( $\left.\Delta G_{\text {alchemical }}^{\text {bulk water }}\right)$. The change in free energy resulting from these transitions is then calculated via thermodynamic integration ${ }^{66}$ and free energy perturbation ${ }^{67}$ methods. The results were validated by checking for microreversibility and the absence of hysteresis ${ }^{56,61}$. Following the completion of the thermodynamic cycle, the standard binding free energy of promoter DNA to the holoenzyme binding pocket was found to be $-6.9 \pm 2.8 \mathrm{kcal} / \mathrm{mol}$.

\section{Molecular dynamics error analysis}

An upper bound on the error of $\Delta G_{\text {restrain }}^{\text {bound }}, \Delta G_{\text {restrain }}^{\text {bulk water }}$ and $\Delta G_{\text {alchemical }}^{\text {bulk water }}$ was determined by the hysteresis between backward and forward simulations ${ }^{33}$. The error in $\Delta G_{\text {alchemical }}^{\text {bound }}$ was determined by performing 3 replicates of the simulation and evaluating the standard deviation (Supplementary Table 4). 


\section{Data availability}

All macromolecular structure data described in this paper have been deposited to the Protein Data Bank and Electron Microscopy Data Bank under the following accession numbers: PDB code 7S00 (AR9 nvRNAP core X-ray structure); PDB code 7S01 (AR9 nvRNAP promoter complex X-ray structure); EMDB code EMD-24765 (AR9 nvRNAP holoenzyme cryo-EM density); EMDB code EMD24763 (AR9 nvRNAP promoter complex cryo-EM density). Publicly available protein atomic models

with the following PDB codes were used in the study: $4 \mathrm{AYB}^{37}, 5 \mathrm{ZX} 3^{43}, 6 \mathrm{C}^{3} \mathrm{Y}^{44}, 5 \mathrm{IPM}^{15}, 6 \mathrm{JBQ}^{16}$, and $7 \mathrm{OGP}^{21}$.

\section{Acknowledgments}

This work was supported by Skoltech NGP Program (Skoltech-MIT joint project), by the Russian Science Foundation (Grant 19-74-00011 to M. L. Sokolova) and by the National Institute of Health (Grant R01GM130942/Subaward 0518GWB837 to S. Borukhov). The work was also supported by the UTMB Department of Biochemistry and Molecular Biology and by the UTMB Sealy Center for Structural Biology and Molecular Biophysics. The MD work was performed using the computing facilities of the Texas Advanced Computing Center (TACC, http://www.tacc.utexas.edu) at The University of Texas for which we are very grateful. We thank the Stanford-SLAC Cryo-EM Facilities, supported by Stanford University, SLAC and the National Institutes of Health S10 Instrumentation Programs that were used to collect the AR9 nvRNAP holoenzyme cryo-EM data. We acknowledge the use of the Advanced Photon Source, a U.S. Department of Energy (DOE) Office of Science User Facility operated for the DOE Office of Science by Argonne National Laboratory under Contract No. DE-AC0206CH11357. We thank the staff of the LS-CAT Sector 21 beamlines that is supported by the Michigan Economic Development Corporation and the Michigan Technology Tri-Corridor (Grant 085P1000817). We acknowledge the use of the Berkeley Center for Structural Biology (supported in part by the Howard 
Hughes Medical Institute) at the Advanced Light Source (a Department of Energy Office of Science User Facility under Contract No. DE-AC02-05CH11231) and we thank the staff of the beamline 5.0.2. Finally, we thank Dr. Mark A. White for his help and assistance with the initial crystallization and Xray data collection of the AR9 nvRNAP core, Dr. Michael B. Sherman for his help with the cryo-EM data collection of all datasets used in this paper, and Dr. Tatyana O. Artamonova for mass-spectrometry analysis of gp226 digestion products. The research reported in this paper extensively used the facilities and resources of the UTMB SCSB Macromolecular Structure X-ray Laboratory and UTMB SCSB CryoEM Laboratory.

\section{Author contributions}

K.V.S. and M.L.S. conceived the study. M.L.S. cloned, purified and crystallized AR9 nvRNAP core, tagless AR9 nvRNAP core, and AR9 nvRNAP holoenzyme in complex with promoter DNA, derivatized crystals, prepared samples for cryo-EM, purified gp226 and performed limited trypsinolisis. A.F. obtained and analyzed all cryo-EM reconstructions, built parts of atomic models, and performed all MD work. A.V.D. purified AR9 nvRNAP holoenzyme and its mutants and performed in vitro transcription assays under the supervision of M.L.S. J.G. under the supervision of M.L.S. crystallized the AR9 nvRNAP holoenzyme in complex with promoter DNA. P.G.L. collected X-ray data, solved all crystal structures, and built and refined all atomic models. The AF team created models of all five AR9

nvRNAP holoenzyme proteins that were used by P.G.L. and A.F. in the interpretation of cryo-EM and X-ray crystallography electron density maps. A.F., M.L.S., S.B., and P.G.L. analyzed the structures. P.G.L. and A.F. wrote the manuscript, which was read, edited, and approved by all authors.

\section{Competing interests}

The authors declare no competing interests. 
Supplementary Information is available for this paper.

\section{Materials and Correspondence}

Correspondence and requests for materials should be addressed to Maria L. Sokolova, Konstantin V. Severinov or Petr G. Leiman. 


\section{Figure Legends}

\section{Figure 1. Organization and promoter consensus of the AR9 nvRNAP.}

a, Organization of the catalytically active core and promoter initiation competent holoenzyme of the AR9 nvRNAP. A pair of genes encode a protein complex that is homologous to the bacterial subunits $\beta$ or $\beta^{\prime}$. The promoter specificity subunit displays no detectable sequence similarity to bacterial sigma factors.

b, The consensus of AR9 late promoters recognized by the nvRNAP. Both DNA strands are shown.

c, The dependance of the AR9 nvRNAP in vitro transcription activity on the position and number of $\mathrm{T}$ bases in the promoter, which is located in the template strand of DNA (bold-underlined).

d, The resistance of recombinantly expressed gp226 to proteolysis by trypsin. The identities and sizes of labeled major products, given as residue ranges, have been established using mass spectrometry. FL stands for the full length protein.

Two technical replicates of two biological replicates of the in vitro transcription and trypsin proteolysis experiments resulted in similar outcomes and one of them is shown. The uncropped autoradiogram and SDS-PAGE are presented in Supplementary Fig. 5.

\section{Fig. 2. Structure of the AR9 nvRNAP promoter complex.}

a, Ribbon diagram of the crystal structure of the AR9 nvRNAP in complex with a forked oligonucleotide containing the AR9 late promoter P077 in its 3'-overhang region. Structural elements that are either unique to the AR9 nvRNAP or common to all RNAPs are labeled and color coded. The $\beta \mathrm{N}$ gp105 subunit is semitransparent for clarity.

b, Schematic of the two oligonucleotides that bound to one AR9 nvRNAP molecule resulting in a transcription bubble-like structure. Bases disordered in the crystal structure are rendered semitransparent. Bases in purple boxes interact with the protein. The dashed lines indicate that the sequence of the non-template strand which is in-register with the promoter is partially complementary to it.

c and $\mathbf{d}$, Structure of the catalytic centers of the AR9 nvRNAP and E. coli RNAP- $\sigma^{\mathrm{S}}$ (PDB code 5IPM ${ }^{15}$ ). Here and elsewhere, TSS stands for the transcription start site. The 2.4 region of $\sigma^{\mathrm{S}}$, which is not present in gp226, is rendered semi-transparent. The E. coli RNAP- $\sigma^{\mathrm{S}}$ structure contains a short RNA product that is not shown for clarity. A part of the DNA non-template strand in the E. coli RNAP- $\sigma^{\mathrm{S}}$ structure is semitransparent for clarity.

Fig. 3. Comparison of the AR9 nvRNAP and $E$. coli RNAP- $\sigma^{\mathrm{E}}$ holoenzymes.

$\mathbf{a}$ and $\mathbf{b}$, Ribbon diagrams of the AR9 nvRNAP and RNAP- $\sigma^{\mathrm{E}}$ (PDB code $6 \mathrm{JBQ}{ }^{16}$ ), respectively, are viewed from the NTP entrance channel. Nucleic acids are not shown for clarity. Gp226 and $\sigma^{\mathrm{E}}$ extend into the plane of the paper and are almost completely obscured by the depth-cueing effect. In both molecules, key elements are colored similarly and labeled. In panel a, the color code is as in Fig. 2 a.

\section{Fig. 4. Structure of the AR9 nvRNAP core.}


a, Molecular surface of the AR9 nvRNAP core crystal structure, with subunits colored as in Fig. 2a, with the downstream DNA oligonucleotide copied from the holoenzyme-promoter structure. The insertion domain partially obstructs the DNA binding cleft.

b, Electrostatic potential is mapped onto the molecular surface of the AR9 nvRNAP core. Both orientations are as in the panel a.

\section{Fig. 5. Cryo-EM structure of the AR9 nvRNAP holoenzyme.}

a, Cryo-EM map of the AR9 nvRNAP holoenzyme contoured at $4.0 \mathrm{std} \mathrm{dev}$ above the mean (semitransparent gray) with the fitted atomic model of the promoter complex (sans DNA) colored as in Fig. 2a.

b, A zoomed-in view of the catalytic cleft demonstrating the degree of gp226 disorder.

\section{Fig. 6. Structure of the promoter specificity subunit gp226.}

a, Ribbon diagram of gp226 with regions structurally similar to bacterial $\sigma$ factors colored in yellow green (helices 2.1 through 2.4 and 4.1) and gold (finger). The unique N- and C-terminal domains colored medium orchid. Residue numbers and identities are given at key locations. The DNA strands are colored as in Fig. 2a and $\mathbf{2 b}$ and are semitransparent. The pseudo ${ }^{-35}$-element binding motif is turquoise and its positively charged and solvent exposed residues are shown in a stick representation.

b, Ribbon diagram of the E. coli $\sigma^{\mathrm{E}}$ factor (PDB code $6 \mathrm{JBQ}^{16}$ ) with its helix-turn-helix motif colored in orange red. Positively charged residues that interact with the DNA are shown in a stick representation and labeled. The DNA backbone is semitransparent.

\section{Fig. 7. Electron density of the AR9 nvRNAP-DNA interacting regions.}

$\mathbf{a}$ and $\mathbf{b}$, Cryo-EM and composite omit X-ray electron density maps of the promoter binding pocket with refined atomic models, respectively. The cryo-EM and X-ray maps are contoured at 5.0 and $1.5 \mathrm{std} \mathrm{dev}$ above the mean, respectively. The carbon atoms are colored as in Fig. 2a. V206, which is critical for U specificity, is colored cyan.

c and d, Cryo-EM and composite omit X-ray electron density maps of the gp226 CTD with refined atomic models, respectively. The cryo-EM and X-ray maps are contoured at 2.0 and $1.0 \mathrm{std}$ dev above the mean, respectively. The ds segment of the downstream oligonucleotide belonging to a neighboring unit cell is shown in the X-ray map (see Supplementary Fig. 1c). Proteins are colored as in Fig. 2a. The pseudo- ${ }^{-35}$ element binding motif is colored light green.

\section{Fig. 8. Interaction of the AR9 nvRNAP with DNA in the promoter complex.}

a, Atomic model of the AR9 nvRNAP promoter recognition element. Gp226 is shown as a semitransparent molecular surface. Only a small fragment of $\beta \mathrm{N}$ gp105 that participates in the formation of the ${ }^{-10} \mathrm{U}$ binding pocket is shown for clarity. Interchain and DNA-intrachain hydrogen bonds are shown as dashed lines. Thin, straight lines connect the C5 atom of the uracil pyrimidine ring to the closest protein or DNA atoms which lie in-plane with the ring. The carbon atoms are colored as in Fig. 2a, except for V206, which is shown in cyan. 
b. The in vitro transcription activity of the AR9 nvRNAP holoenzyme containing wild type (WT) gp226 or the V206G gp226 mutant have been tested using various U- and T-containing templates.

c, Interaction of the upstream oligonucleotide with the gp226 NTD. The same gp226 and $\beta \mathrm{N}$ gp105 fragments as in Fig. 8a are shown but are tilted to improve visibility. The nucleotides are numbered according to the original nomenclature of the template strand (see Fig. 2b). The putative base identities and their numbers relative to the TSS (as would be found in a dsDNA transcription bubble) are given in midnight blue colored boxes.

d, The in vitro transcription activity of the AR9 nvRNAP holoenzyme containing gp226 mutants with an altered structure of the non-template strand binding groove.

e and $\mathbf{f}$, The surface electrostatic potentials of the pseudo ${ }^{-35}$-element binding motif in WT gp226 and the A gp226 mutant.

g, The ssDNA and dsDNA in vitro transcription activities of the AR9 nvRNAP holoenzyme containing WT gp226 and the $\mathrm{A}^{5}$ gp226 mutant.

For each in vitro transcription experiment, two technical replicates of two biological replicates resulted in similar outcomes and one of them is shown. The uncropped autoradiograms are presented in Supplementary Fig. 5.

Fig. 9. Derivation of the promoter binding free energy using molecular dynamics.

a, Thermodynamic cycle of promoter binding. The PPC superscript (e.g. DNA ${ }^{\mathrm{PPC}}$ ) stands for Promoter Pocket Conformation in regard to the structure of the $3^{\prime}-{ }^{-11} \mathrm{UUG}^{-9}-5^{\prime}$ DNA trinucleotide.

b, Equilibration and relaxation of the cryo-EM derived atomic model of the AR9 nvRNAP holoenzyme with the $3^{\prime}-{ }^{-11} U_{U G}{ }^{-9}-5^{\prime}$ DNA trinucleotide bound to the promoter pocket.

c, Equilibration and relaxation of the $3^{\prime}-{ }^{-11} \mathrm{UUG}^{-9}-5^{\prime}$ trinucleotide in bulk water.

$\mathbf{d}$ and $\mathbf{e}$, Energetics of forward and backward alchemical transformations of the $3^{\prime}-{ }^{-11} \mathrm{UUG}^{-9}-5^{\prime}$ DNA trinucleotide in the promoter pocket of the AR9 nvRNAP holoenzyme and in the PPC in bulk water, respectively.

$\mathbf{f}, \mathbf{g}, \mathbf{h}, \mathbf{i}, \mathbf{j}, \mathbf{k}, \mathbf{l}$, Entropic cost of applying seven harmonic constraints to the $3^{\prime}-{ }^{-11} \mathrm{UUG}^{-9}-5^{\prime}$ DNA trinucleotide to maintain it in the promoter pocket-bound state.

$\mathbf{m}$, Entropic cost of the harmonic RMSD constraint on the $3^{\prime}-{ }^{-11} U_{U G}{ }^{-9}-5^{\prime}$ DNA trinucleotide to maintain the PPC.

Fig. 10. The mechanism of template strand promoter recognition in dsDNA.

For clarity, both proteins comprising the $\mathrm{N}$ - and $\mathrm{C}$-terminal parts of the $\beta$ and $\beta^{\prime}$ subunits are shown in the same color (light yellow for $\beta$ and light green for $\beta^{\prime}$ ). The putatively degenerate, third position base ${ }^{-9} \mathrm{C}$ of the promoter-complementary motif ${ }^{-11} \mathrm{AA}(\mathrm{C}) \mathrm{A}^{-8}$ is shown as a semitransparent rod. The pluses indicate positively charged elements of the gp226 NTD and CTD molecular surface that participate in DNA binding. See the main text for full explanation. 


\section{References}

1. Lavysh, D., Sokolova, M., Minakhin, L., Yakunina, M., Artamonova, T., Kozyavkin, S., Makarova, K.S., Koonin, E.V. \& Severinov, K. The genome of AR9, a giant transducing Bacillus phage encoding two multisubunit RNA polymerases. Virology 495, 185-96 (2016).

2. Lavysh, D., Sokolova, M., Slashcheva, M., Forstner, K.U. \& Severinov, K. Transcription Profiling of Bacillus subtilis Cells Infected with AR9, a Giant Phage Encoding Two Multisubunit RNA Polymerases. mBio 8(2017).

3. Sokolova, M., Borukhov, S., Lavysh, D., Artamonova, T., Khodorkovskii, M. \& Severinov, K. A non-canonical multisubunit RNA polymerase encoded by the AR9 phage recognizes the template strand of its uracil-containing promoters. Nucleic Acids Res 45, 5958-5967 (2017).

4. Sokolova, M.L., Misovetc, I. \& K, V.S. Multisubunit RNA Polymerases of Jumbo Bacteriophages. Viruses 12(2020).

5. Korn, A.M., Hillhouse, A.E., Sun, L. \& Gill, J.J. Comparative Genomics of Three Novel Jumbo Bacteriophages Infecting Staphylococcus aureus. J Virol, JVI0239120 (2021).

6. Skurnik, M., Hyytiainen, H.J., Happonen, L.J., Kiljunen, S., Datta, N., Mattinen, L., Williamson, K., Kristo, P., Szeliga, M., Kalin-Manttari, L., Ahola-Iivarinen, E., Kalkkinen, N. \& Butcher, S.J. Characterization of the genome, proteome, and structure of yersiniophage phiR1-37. J Virol 86, 12625-42 (2012).

7. Bae, B., Feklistov, A., Lass-Napiorkowska, A., Landick, R. \& Darst, S.A. Structure of a bacterial RNA polymerase holoenzyme open promoter complex. Elife 4(2015).

8. Ishihama, A. Subunit of assembly of Escherichia coli RNA polymerase. Adv Biophys 14, 1-35 (1981).

9. Lane, W.J. \& Darst, S.A. Molecular evolution of multisubunit RNA polymerases: structural analysis. J Mol Biol 395, 686-704 (2010).

10. Minakhin, L., Bhagat, S., Brunning, A., Campbell, E.A., Darst, S.A., Ebright, R.H. \& Severinov, K. Bacterial RNA polymerase subunit omega and eukaryotic RNA polymerase subunit RPB6 are sequence, structural, and functional homologs and promote RNA polymerase assembly. Proc Natl Acad Sci U S A 98, 892-7 (2001).

11. Lee, J. \& Borukhov, S. Bacterial RNA Polymerase-DNA Interaction-The Driving Force of Gene Expression and the Target for Drug Action. Front Mol Biosci 3, 73 (2016).

12. Kuznedelov, K., Korzheva, N., Mustaev, A. \& Severinov, K. Structure-based analysis of RNA polymerase function: the largest subunit's rudder contributes critically to elongation complex stability and is not involved in the maintenance of RNA-DNA hybrid length. EMBOJ 21, 136978 (2002).

13. Chlenov, M., Masuda, S., Murakami, K.S., Nikiforov, V., Darst, S.A. \& Mustaev, A. Structure and function of lineage-specific sequence insertions in the bacterial RNA polymerase beta' subunit. J Mol Biol 353, 138-54 (2005).

14. Bao, Y.\& Landick, R. Obligate movements of an active site-linked surface domain control RNA polymerase elongation and pausing via a Phe-pocket anchor. bioRxiv, 2021.01.27.428476 (2021).

15. Liu, B., Zuo, Y. \& Steitz, T.A. Structures of E. coli sigmaS-transcription initiation complexes provide new insights into polymerase mechanism. Proc Natl Acad Sci US A 113, 4051-6 (2016).

16. Fang, C., Li, L., Shen, L., Shi, J., Wang, S., Feng, Y. \& Zhang, Y. Structures and mechanism of transcription initiation by bacterial ECF factors. Nucleic Acids Res 47, 7094-7104 (2019). 
17. Feklistov, A. \& Darst, S.A. Structural basis for promoter-10 element recognition by the bacterial RNA polymerase sigma subunit. Cell 147, 1257-69 (2011).

18. Feklistov, A., Sharon, B.D., Darst, S.A. \& Gross, C.A. Bacterial sigma factors: a historical, structural, and genomic perspective. Annu Rev Microbiol 68, 357-76 (2014).

19. Paget, M.S. Bacterial Sigma Factors and Anti-Sigma Factors: Structure, Function and Distribution. Biomolecules 5, 1245-65 (2015).

20. Holm, L. \& Laakso, L.M. Dali server update. Nucleic Acids Res 44, W351-5 (2016).

21. de, Y.G.N., Orekhova, M., Lai Wan Loong, Y.T.E., Litvinova, A., Ramlaul, K., Artamonova, T., Melnikov, A.S., Serdobintsev, P., Aylett, C.H.S. \& Yakunina, M. Structure of the bacteriophage PhiKZ non-virion RNA polymerase. Nucleic Acids Res 49, $7732-7739$ (2021).

22. Yakunina, M., Artamonova, T., Borukhov, S., Makarova, K.S., Severinov, K. \& Minakhin, L. A non-canonical multisubunit RNA polymerase encoded by a giant bacteriophage. Nucleic Acids Res 43, 10411-20 (2015).

23. Jumper, J., Evans, R., Pritzel, A., Green, T., Figurnov, M., Ronneberger, O., Tunyasuvunakool, K., Bates, R., Zidek, A., Potapenko, A., Bridgland, A., Meyer, C., Kohl, S.A.A., Ballard, A.J., Cowie, A., Romera-Paredes, B., Nikolov, S., Jain, R., Adler, J., Back, T., Petersen, S., Reiman, D., Clancy, E., Zielinski, M., Steinegger, M., Pacholska, M., Berghammer, T., Bodenstein, S., Silver, D., Vinyals, O., Senior, A.W., Kavukcuoglu, K., Kohli, P. \& Hassabis, D. Highly accurate protein structure prediction with AlphaFold. Nature (2021).

24. Mariani, V., Biasini, M., Barbato, A. \& Schwede, T. IDDT: a local superposition-free score for comparing protein structures and models using distance difference tests. Bioinformatics 29, 2722-8 (2013).

25. Brennan, R.G. \& Matthews, B.W. The helix-turn-helix DNA binding motif. J Biol Chem 264, 1903-6 (1989).

26. Campbell, E.A., Muzzin, O., Chlenov, M., Sun, J.L., Olson, C.A., Weinman, O., Trester-Zedlitz, M.L. \& Darst, S.A. Structure of the bacterial RNA polymerase promoter specificity sigma subunit. Mol Cell 9, 527-39 (2002).

27. Rohs, R., Jin, X., West, S.M., Joshi, R., Honig, B. \& Mann, R.S. Origins of specificity in proteinDNA recognition. Annu Rev Biochem 79, 233-69 (2010).

28. Ceyssens, P.J., Minakhin, L., Van den Bossche, A., Yakunina, M., Klimuk, E., Blasdel, B., De Smet, J., Noben, J.P., Blasi, U., Severinov, K. \& Lavigne, R. Development of giant bacteriophage phiKZ is independent of the host transcription apparatus. $J$ Virol 88, 10501-10 (2014).

29. Mesyanzhinov, V.V., Robben, J., Grymonprez, B., Kostyuchenko, V.A., Bourkaltseva, M.V., Sykilinda, N.N., Krylov, V.N. \& Volckaert, G. The genome of bacteriophage phiKZ of Pseudomonas aeruginosa. J Mol Biol 317, 1-19 (2002).

30. Chaikeeratisak, V., Nguyen, K., Egan, M.E., Erb, M.L., Vavilina, A. \& Pogliano, J. The Phage Nucleus and Tubulin Spindle Are Conserved among Large Pseudomonas Phages. Cell Rep 20, 1563-1571 (2017).

31. Woo, H.J. \& Roux, B. Calculation of absolute protein-ligand binding free energy from computer simulations. Proc Natl Acad Sci U S A 102, 6825-30 (2005).

32. Gumbart, J., Roux, B. \& Chipot, C. Protein: ligand standard binding free energies: A tutorial for alchemical and geometrical transformations. University of Illinois at Urbana-Champaign, USA (2011).

33. Gumbart, J.C., Roux, B. \& Chipot, C. Standard binding free energies from computer simulations: What is the best strategy? J Chem Theory Comput 9, 794-802 (2013). 
34. Phillips, J.C., Braun, R., Wang, W., Gumbart, J., Tajkhorshid, E., Villa, E., Chipot, C., Skeel, R.D., Kale, L. \& Schulten, K. Scalable molecular dynamics with NAMD. J Comput Chem 26, 1781-802 (2005).

35. Vonrhein, C., Blanc, E., Roversi, P. \& Bricogne, G. Automated structure solution with autoSHARP. Methods Mol Biol 364, 215-30 (2007).

36. Zimmermann, L., Stephens, A., Nam, S.Z., Rau, D., Kubler, J., Lozajic, M., Gabler, F., Soding, J., Lupas, A.N. \& Alva, V. A Completely Reimplemented MPI Bioinformatics Toolkit with a New HHpred Server at its Core. J Mol Biol 430, 2237-2243 (2018).

37. Wojtas, M.N., Mogni, M., Millet, O., Bell, S.D. \& Abrescia, N.G. Structural and functional analyses of the interaction of archaeal RNA polymerase with DNA. Nucleic Acids Res 40, 994152 (2012).

38. Emsley, P. \& Cowtan, K. Coot: model-building tools for molecular graphics. Acta Crystallogr D Biol Crystallogr 60, 2126-32 (2004).

39. Rossmann, M.G. The Molecular Replacement Method. A Collection of Papers on the Use of Non-Crystallographic Symmetry., (Gordon and Breach, New York, 1972).

40. McCoy, A.J., Grosse-Kunstleve, R.W., Adams, P.D., Winn, M.D., Storoni, L.C. \& Read, R.J. Phaser crystallographic software. Journal of applied crystallography 40, 658-674 (2007).

41. Cowtan, K. Recent developments in classical density modification. Acta crystallographica. Section D, Biological crystallography 66, 470-8 (2010).

42. Cowtan, K. The Buccaneer software for automated model building. 1. Tracing protein chains. Acta Crystallogr D Biol Crystallogr 62, 1002-11 (2006).

43. Li, L., Fang, C., Zhuang, N., Wang, T. \& Zhang, Y. Structural basis for transcription initiation by bacterial ECF sigma factors. Nat Commun 10, 1153 (2019).

44. Narayanan, A., Vago, F.S., Li, K., Qayyum, M.Z., Yernool, D., Jiang, W. \& Murakami, K.S. Cryo-EM structure of Escherichia coli sigma(70) RNA polymerase and promoter DNA complex revealed a role of sigma non-conserved region during the open complex formation. $J$ Biol Chem 293, 7367-7375 (2018).

45. Adams, P.D., Afonine, P.V., Bunkoczi, G., Chen, V.B., Echols, N., Headd, J.J., Hung, L.W., Jain, S., Kapral, G.J., Grosse Kunstleve, R.W., McCoy, A.J., Moriarty, N.W., Oeffner, R.D., Read, R.J., Richardson, D.C., Richardson, J.S., Terwilliger, T.C. \& Zwart, P.H. The Phenix software for automated determination of macromolecular structures. Methods 55, 94-106 (2011).

46. Kryshtafovych, A., Moult, J., Albrecht, R., Chang, G., Chao, K., Fraser, A., Greenfield, J., Hartmann, M., Herzberg, O., Josts, I., Leiman, P., Linden, S., Lupas, A., Nelson, D., Rees, S., Shang, X., Sokolova, M. \& Tidow, H. Computational models in the service of X-ray and cryoEM structure determination. (Authorea Preprints, 2021).

47. Tang, G., Peng, L., Baldwin, P.R., Mann, D.S., Jiang, W., Rees, I. \& Ludtke, S.J. EMAN2: an extensible image processing suite for electron microscopy. J Struct Biol 157, 38-46 (2007).

48. Zivanov, J., Nakane, T., Forsberg, B.O., Kimanius, D., Hagen, W.J., Lindahl, E. \& Scheres, S.H. New tools for automated high-resolution cryo-EM structure determination in RELION-3. Elife 7(2018).

49. Punjani, A., Rubinstein, J.L., Fleet, D.J. \& Brubaker, M.A. cryoSPARC: algorithms for rapid unsupervised cryo-EM structure determination. Nat Methods 14, 290-296 (2017).

50. Zheng, S.Q., Palovcak, E., Armache, J.P., Verba, K.A., Cheng, Y. \& Agard, D.A. MotionCor2: anisotropic correction of beam-induced motion for improved cryo-electron microscopy. Nat Methods 14, 331-332 (2017). 
51. Rohou, A. \& Grigorieff, N. CTFFIND4: Fast and accurate defocus estimation from electron micrographs. J Struct Biol 192, 216-21 (2015).

52. Punjani, A., Zhang, H. \& Fleet, D.J. Non-uniform refinement: adaptive regularization improves single-particle cryo-EM reconstruction. Nat Methods 17, 1214-1221 (2020).

53. Zhang, K. Gctf: Real-time CTF determination and correction. J Struct Biol 193, 1-12 (2016).

54. Huang, J. \& MacKerell, A.D., Jr. CHARMM36 all-atom additive protein force field: validation based on comparison to NMR data. J Comput Chem 34, 2135-45 (2013).

55. Feller, S.E., Zhang, Y., Pastor, R.W. \& Brooks, B.R. Constant pressure molecular dynamics simulation: The Langevin piston method. The Journal of Chemical Physics 103, 4613-4621 (1995).

56. Pohorille, A., Jarzynski, C. \& Chipot, C. Good practices in free-energy calculations. J Phys Chem $B$ 114, 10235-53 (2010).

57. Florin, G., Klein, M.L. \& Hénin, J. Using collective variables to drive molecular dynamics simulations. Molecular Physics 111, 3345-3362 (2013).

58. Darden, T., York, D. \& Pedersen, L. Particle mesh Ewald: An N·log(N) method for Ewald sums in large systems. The Journal of Chemical Physics 98, 10089-10092 (1993).

59. Beutler, T.C., Mark, A.E., van Schaik, R.C., Gerber, P.R. \& van Gunsteren, W.F. Avoiding singularities and numerical instabilities in free energy calculations based on molecular simulations. Chemical Physics Letters 222, 529-539 (1994).

60. Zacharias, M., Straatsma, T.P. \& McCammon, J.A. Separation-shifted scaling, a new scaling method for Lennard-Jones interactions in thermodynamic integration. The Journal of Chemical Physics 100, 9025-9031 (1994).

61. Bennett, C.H. Efficient estimation of free energy differences from Monte Carlo data. Journal of Computational Physics 22, 245-268 (1976).

62. Humphrey, W., Dalke, A. \& Schulten, K. VMD: visual molecular dynamics. J Mol Graph 14, 33-8, 27-8 (1996).

63. Harrach, M.F. \& Drossel, B. Structure and dynamics of TIP3P, TIP4P, and TIP5P water near smooth and atomistic walls of different hydroaffinity. The Journal of Chemical Physics 140, 174501 (2014).

64. Roux, B., Nina, M., Pomes, R. \& Smith, J.C. Thermodynamic stability of water molecules in the bacteriorhodopsin proton channel: a molecular dynamics free energy perturbation study. Biophys J 71, 670-81 (1996).

65. Gilson, M.K., Given, J.A., Bush, B.L. \& McCammon, J.A. The statistical-thermodynamic basis for computation of binding affinities: a critical review. Biophys $J$ 72, 1047-69 (1997).

66. Frenkel, D. \& Smit, B. Free Energy Calculations. in Understanding Molecular Simulation (Second Edition) (eds. Frenkel, D. \& Smit, B.) 167-200 (Academic Press, 2002).

67. Beveridge, D.L. \& DiCapua, F.M. Free energy via molecular simulation: applications to chemical and biomolecular systems. Annu Rev Biophys Biophys Chem 18, 431-92 (1989). 


\section{Figures}

a

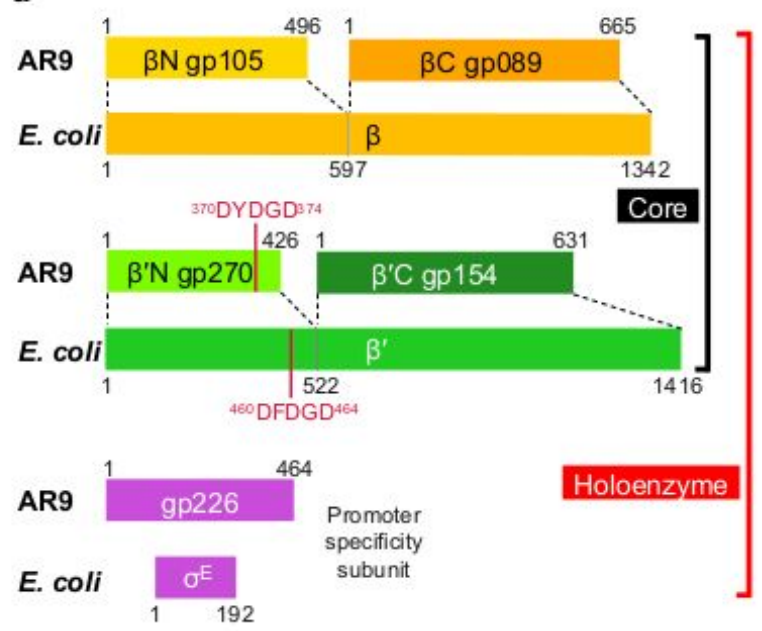

c

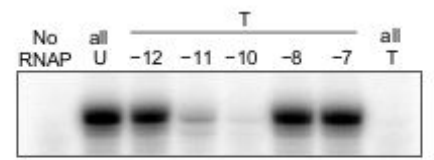

atcacatattggag-3 allu 3 -gttauUUGUatat aTa tagtgtataacct c-5

atcacatat tggag-3

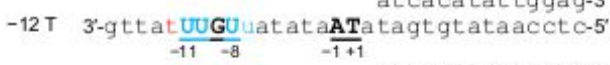

-11 T 3-gtta TUGU $\frac{\text { TU }}{-11-8} \underset{-1+1}{\text { ATa }}$ tagtgtataacctc-5' at caca tat tggag-3 -10 T 3 -gttauUTGU Uatat $\underset{-11+1}{\mathbf{A T}}$ tagtgtataacet C-5

atcacatat tggag-3 -8 T 3-gttauUUGT atcacatat tggag-3 -7 T 3-gttauUUGU tatat a ATa tagtgtataacct c-5 atca ca tat tggag-3 all $3^{\prime}$-gttat $\frac{\text { TTGT tatataATa }}{-11-8}$ tagtgtataacctc-5' b

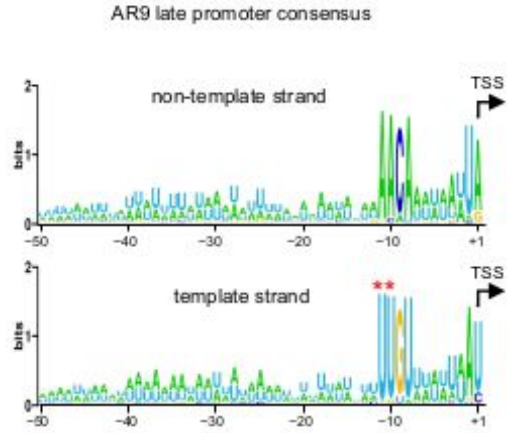

d

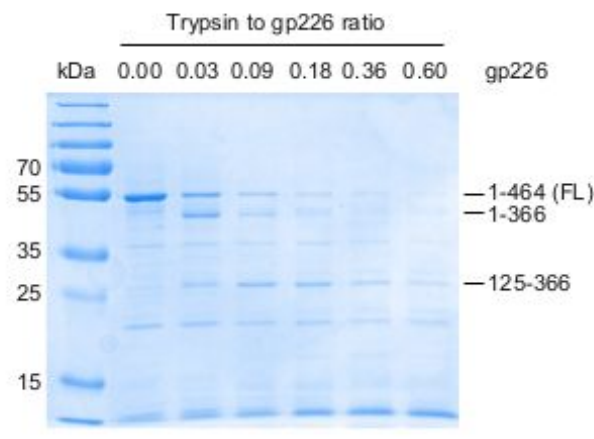

\section{Figure 1}

\section{Figure 1}

Organization and promoter consensus of the AR9 nvRNAP. a, Organization of the catalytically active core and promoter initiation competent holoenzyme of the AR9 nvRNAP. A pair of genes encode a protein complex that is homologous to the bacterial subunits $\beta$ or $\beta^{\prime}$. The promoter specificity subunit displays no 
detectable sequence similarity to bacterial sigma factors. b, The consensus of AR9 late promoters recognized by the nvRNAP. Both DNA strands are shown. c, The dependance of the AR9 nvRNAP in vitro transcription activity on the position and number of $\mathrm{T}$ bases in the promoter, which is located in the template strand of DNA (bold-underlined). $d$, The resistance of recombinantly expressed gp226 to proteolysis by trypsin. The identities and sizes of labeled major products, given as residue ranges, have been established using mass spectrometry. FL stands for the full length protein. Two technical replicates of two biological replicates of the in vitro transcription and trypsin proteolysis experiments resulted in similar outcomes and one of them is shown. The uncropped autoradiogram and SDS-PAGE are presented in Supplementary Fig. 5. 
a

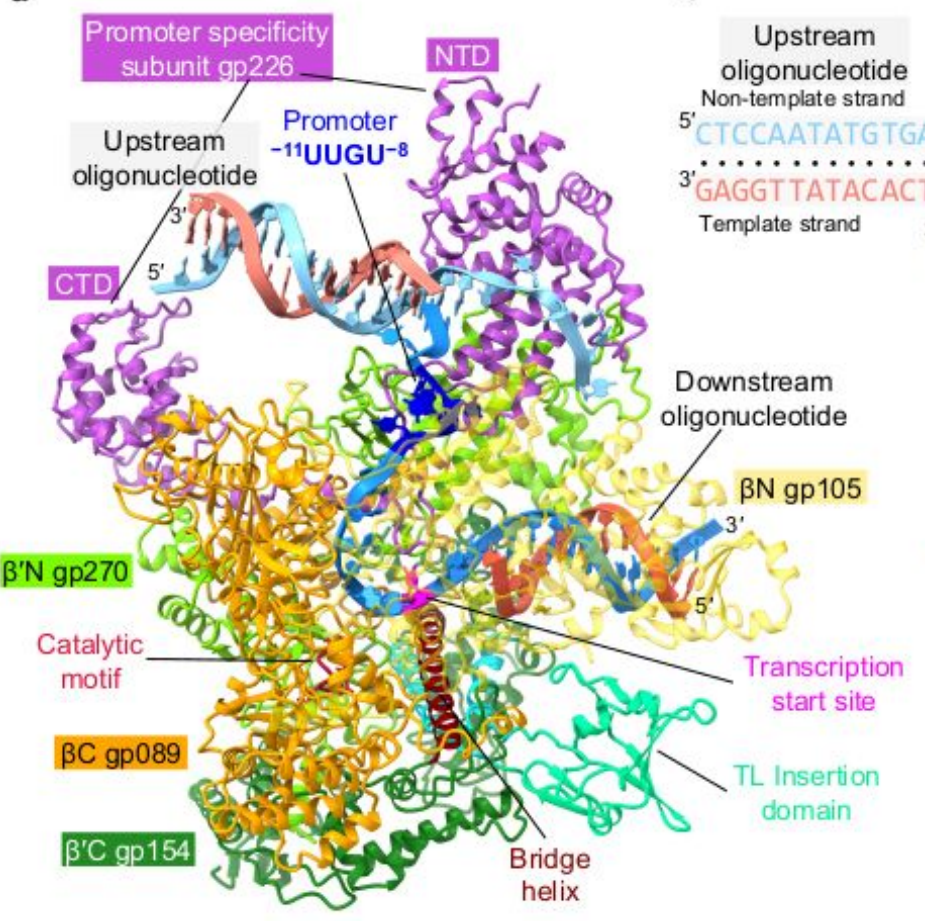

\section{b}

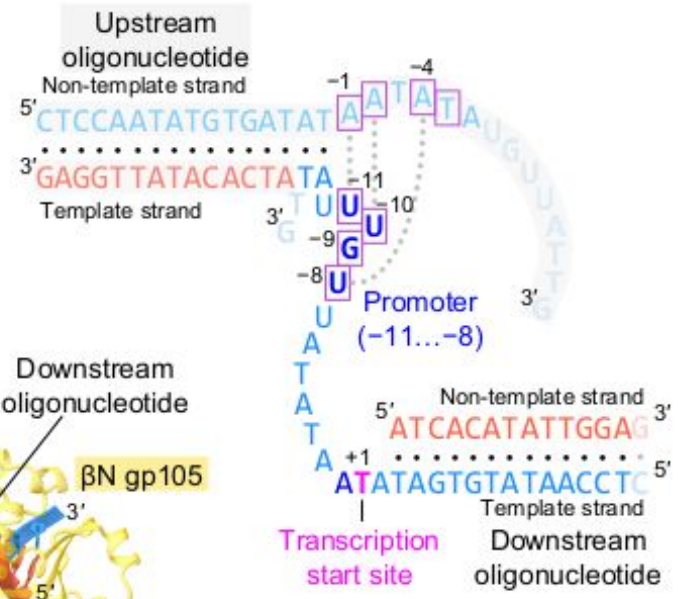

c

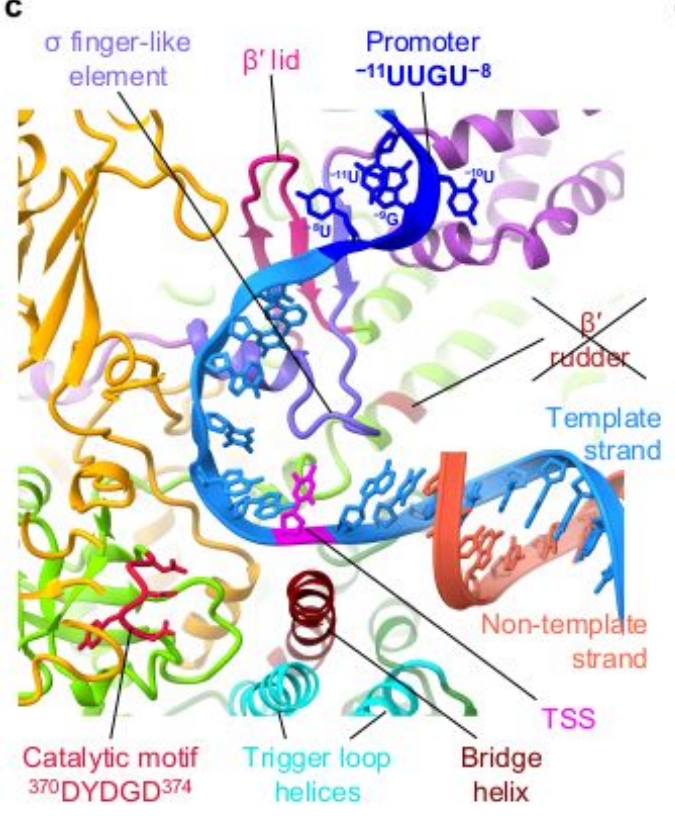

d

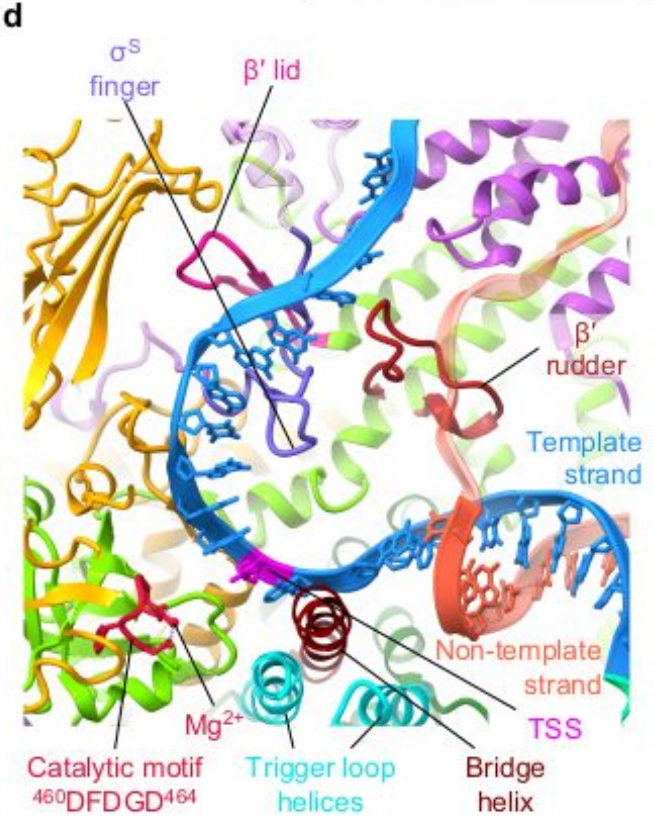

Figure 2

\section{Figure 2}

Structure of the AR9 nvRNAP promoter complex. a, Ribbon diagram of the crystal structure of the AR9 nvRNAP in complex with a forked oligonucleotide containing the AR9 late promoter P077 in its 3'overhang region. Structural elements that are either unique to the AR9 nvRNAP or common to all RNAPs are labeled and color coded. The $\beta \mathrm{N}$ gp105 subunit is semitransparent for clarity. b, Schematic of the two oligonucleotides that bound to one AR9 nvRNAP molecule resulting in a transcription bubble-like 
structure. Bases disordered in the crystal structure are rendered semitransparent. Bases in purple boxes interact with the protein. The dashed lines indicate that the sequence of the non-template strand which is in-register with the promoter is partially complementary to it. $c$ and d, Structure of the catalytic centers of the AR9 nvRNAP and E. coli RNAP- $\sigma S$ (PDB code 5IPM15). Here and elsewhere, TSS stands for the transcription start site. The 2.4 region of $\sigma S$, which is not present in gp226, is rendered semi-transparent. The E. coli RNAP- $\sigma S$ structure contains a short RNA product that is not shown for clarity. A part of the DNA non-template strand in the E. coli RNAP-oS structure is semitransparent for clarity.
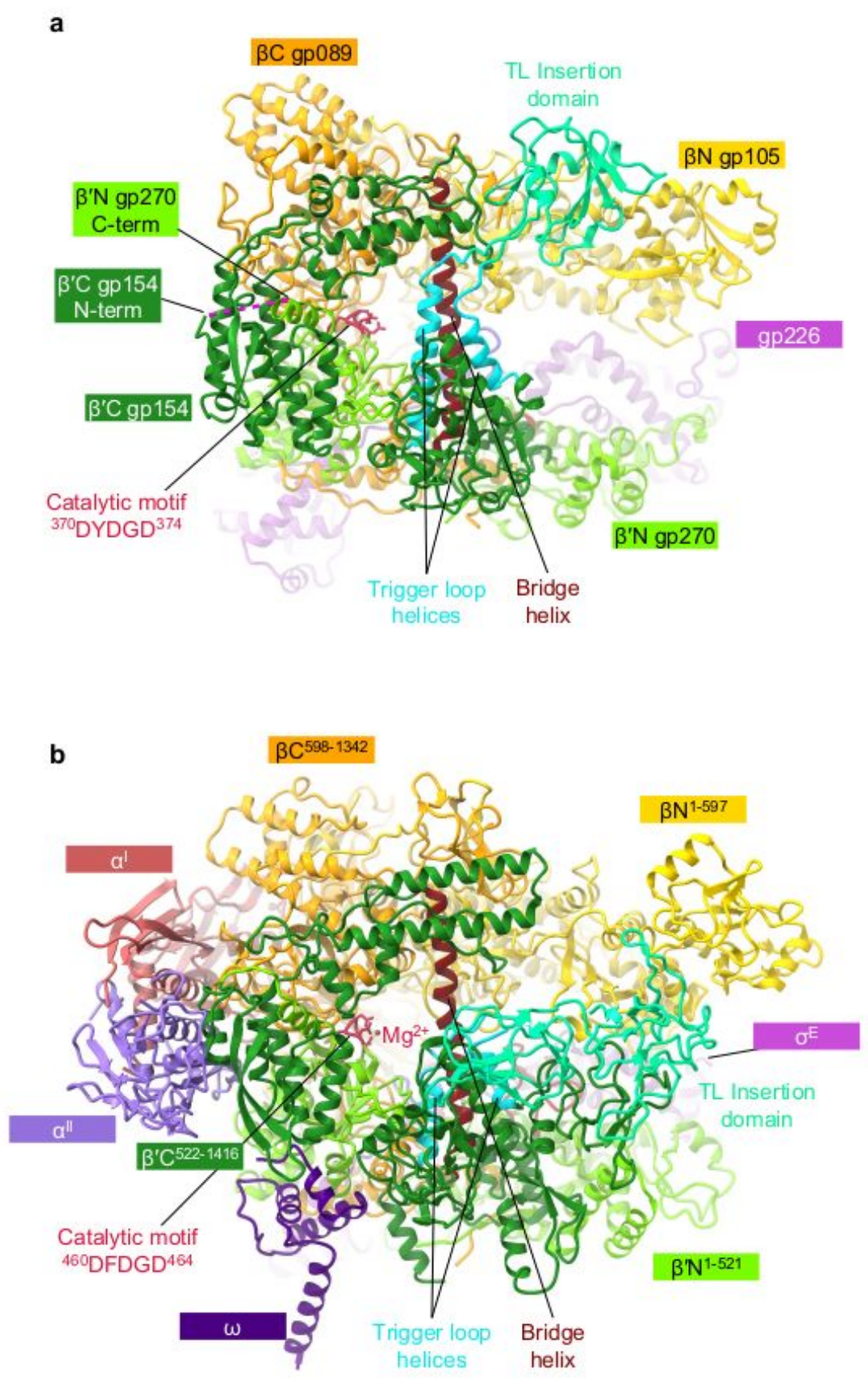

Figure 3 


\section{Figure 3}

Comparison of the AR9 nvRNAP and E. coli RNAP- $\sigma E$ holoenzymes. $a$ and b, Ribbon diagrams of the AR9 nvRNAP and RNAP- $\sigma E$ (PDB code 6JBQ16), respectively, are viewed from the NTP entrance channel. Nucleic acids are not shown for clarity. Gp226 and $\sigma E$ extend into the plane of the paper and are almost completely obscured by the depth-cueing effect. In both molecules, key elements are colored similarly and labeled. In panel a, the color code is as in Fig. 2a.
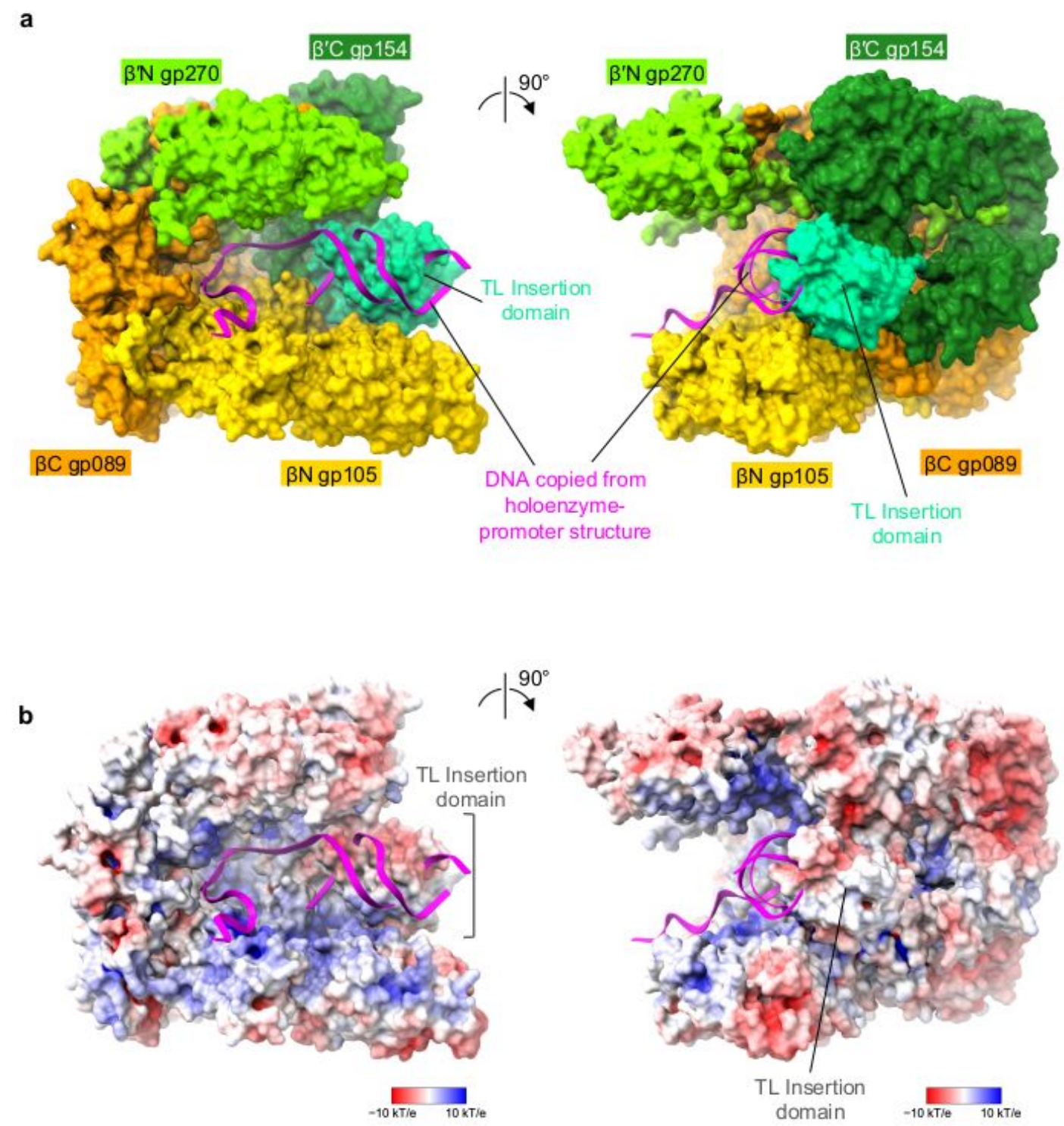

Figure 4 


\section{Figure 4}

Structure of the AR9 nvRNAP core. a, Molecular surface of the AR9 nvRNAP core crystal structure, with subunits colored as in Fig. 2a, with the downstream DNA oligonucleotide copied from the holoenzymepromoter structure. The insertion domain partially obstructs the DNA binding cleft. b, Electrostatic potential is mapped onto the molecular surface of the AR9 nvRNAP core. Both orientations are as in the panel a.

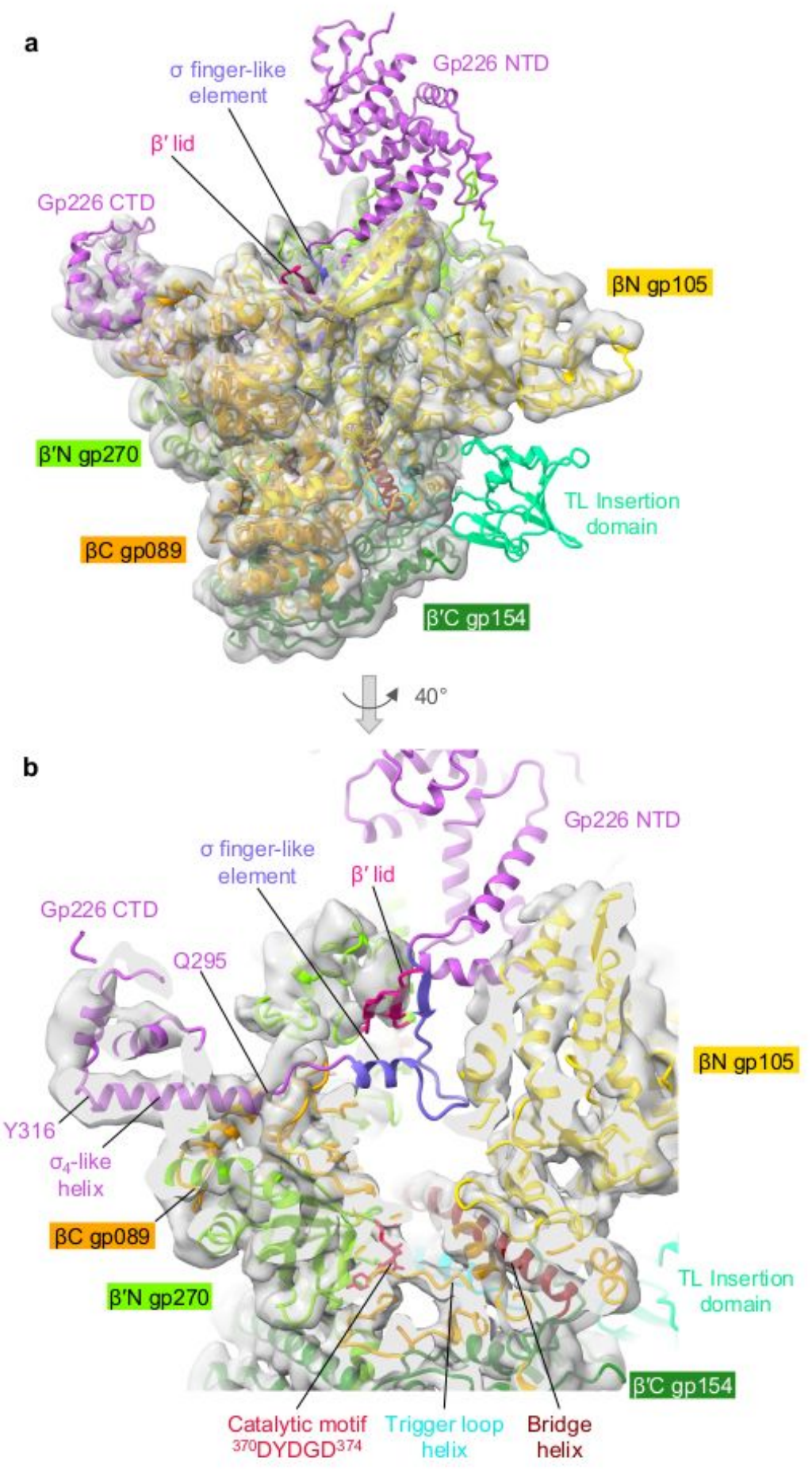

Figure 5 
Figure 5

Cryo-EM structure of the AR9 nvRNAP holoenzyme. a, Cryo-EM map of the AR9 nvRNAP holoenzyme contoured at 4.0 std dev above the mean (semitransparent gray) with the fitted atomic model of the promoter complex (sans DNA) colored as in Fig. 2a. b, A zoomed-in view of the catalytic cleft demonstrating the degree of gp226 disorder.
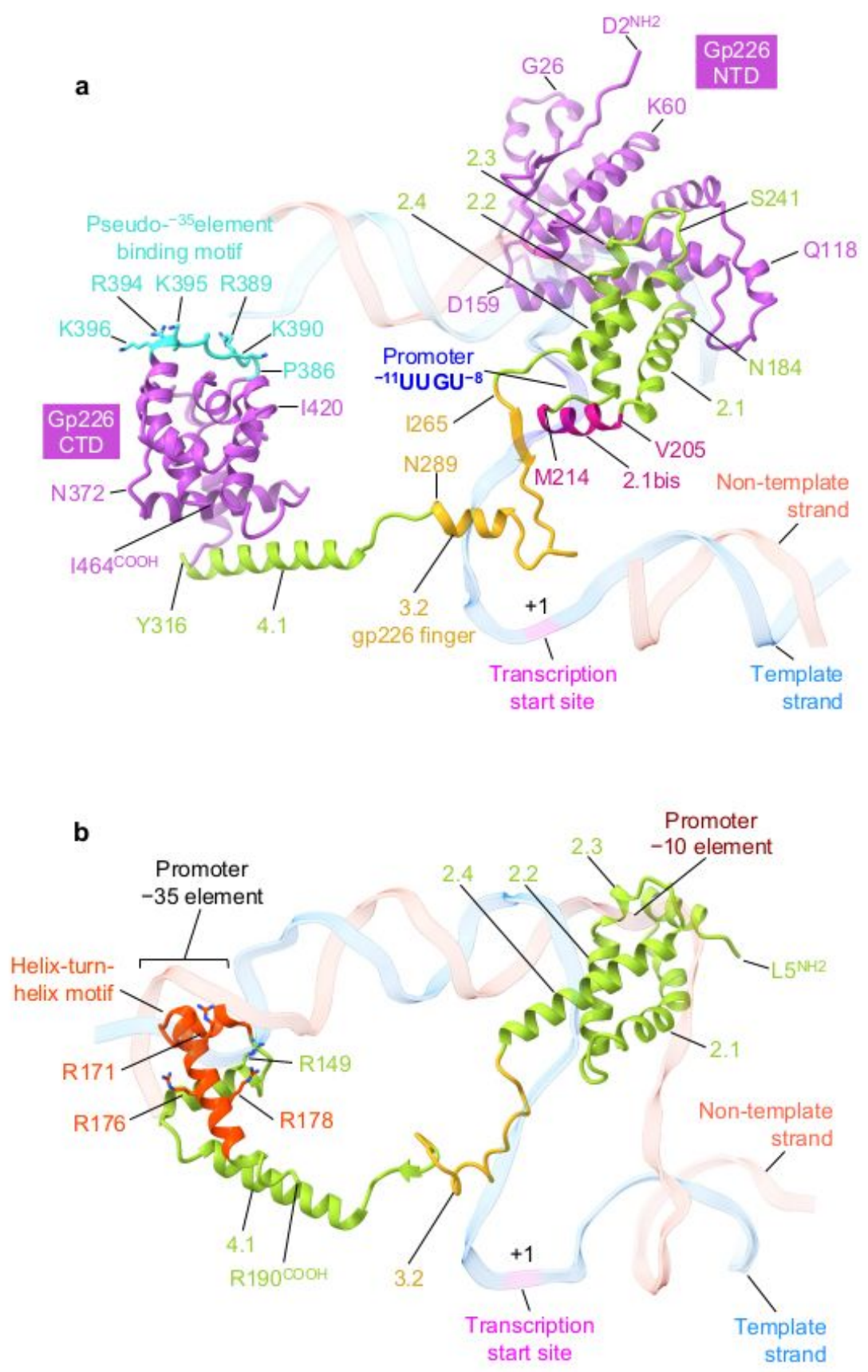

Figure 6

Figure 6 
Structure of the promoter specificity subunit gp226. a, Ribbon diagram of gp226 with regions structurally similar to bacterial $\sigma$ factors colored in yellow green (helices 2.1 through 2.4 and 4.1) and gold (finger). The unique $\mathrm{N}$ - and $\mathrm{C}$-terminal domains colored medium orchid. Residue numbers and identities are given at key locations. The DNA strands are colored as in Fig. $2 a$ and $2 b$ and are semitransparent. The pseudo-35-element binding motif is turquoise and its positively charged and solvent exposed residues are shown in a stick representation. b, Ribbon diagram of the E. coli $\sigma E$ factor (PDB code 6JBQ16) with its helix-turn-helix motif colored in orange red. Positively charged residues that interact with the DNA are shown in a stick representation and labeled. The DNA backbone is semitransparent. 

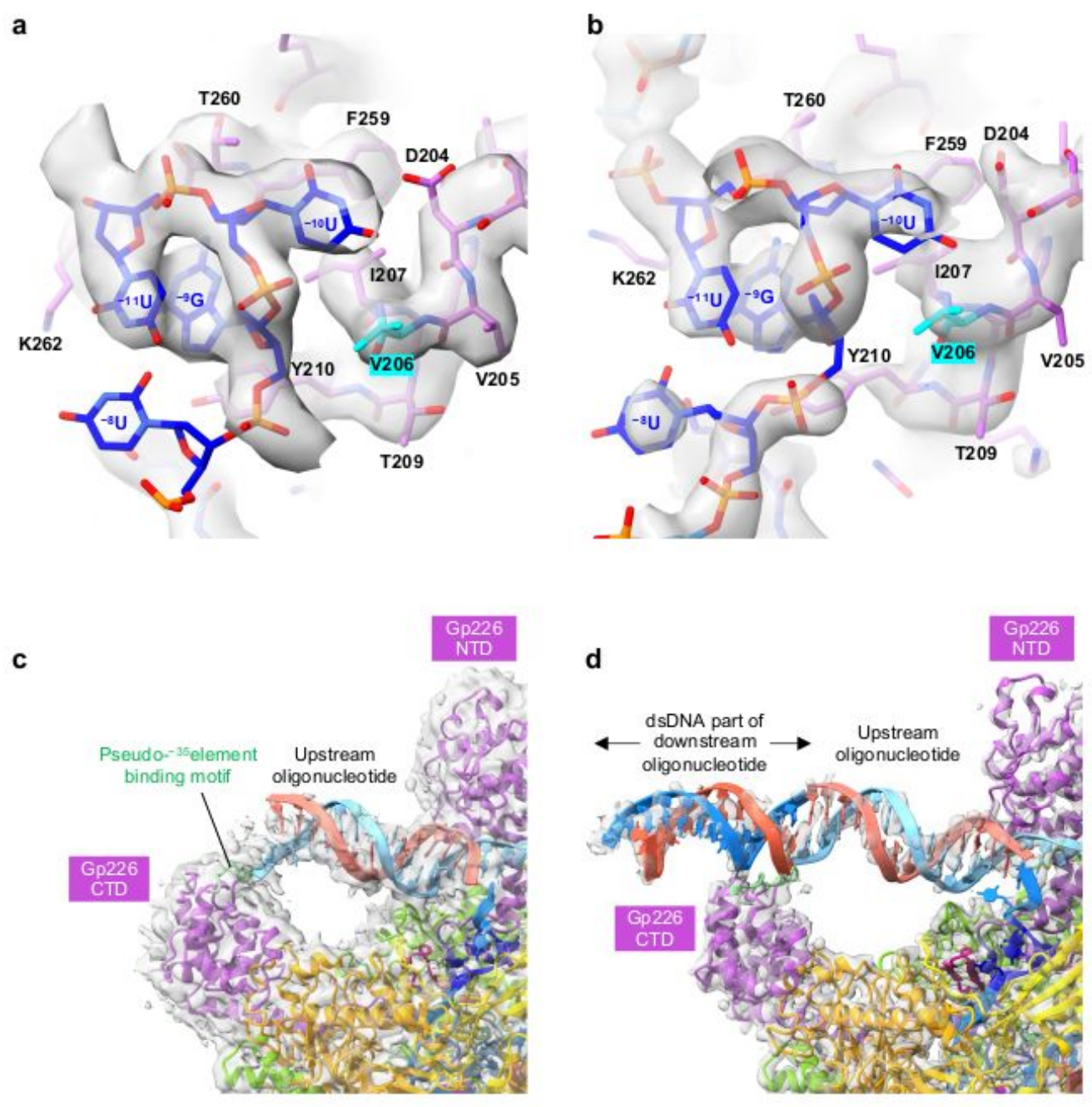

Figure 7

\section{Figure 7}

Electron density of the AR9 nvRNAP-DNA interacting regions. a and b, Cryo-EM and composite omit X-ray electron density maps of the promoter binding pocket with refined atomic models, respectively. The cryoEM and X-ray maps are contoured at 5.0 and 1.5 std dev above the mean, respectively. The carbon atoms are colored as in Fig. 2a. V206, which is critical for U specificity, is colored cyan. c and d, Cryo-EM and composite omit X-ray electron density maps of the gp226 CTD with refined atomic models, respectively. 
The cryo-EM and X-ray maps are contoured at 2.0 and $1.0 \mathrm{std}$ dev above the mean, respectively. The ds segment of the downstream oligonucleotide belonging to a neighboring unit cell is shown in the X-ray map (see Supplementary Fig. 1c). Proteins are colored as in Fig. 2a. The pseudo--35element binding motif is colored light green.

a

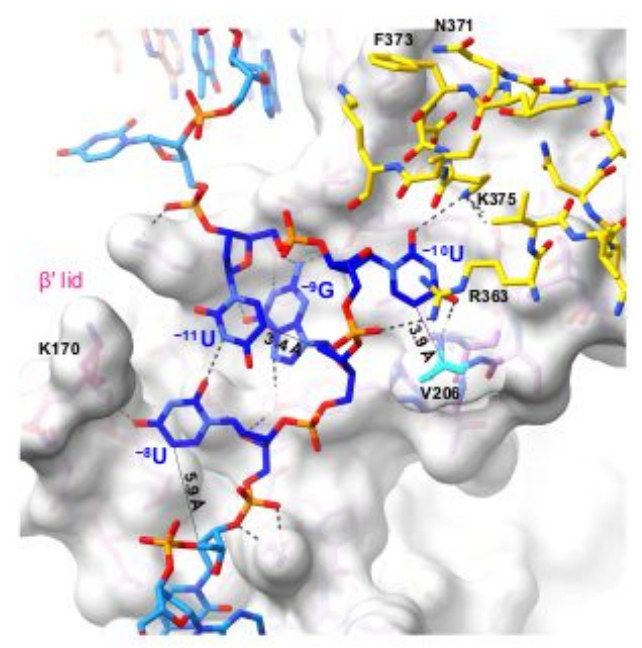

c

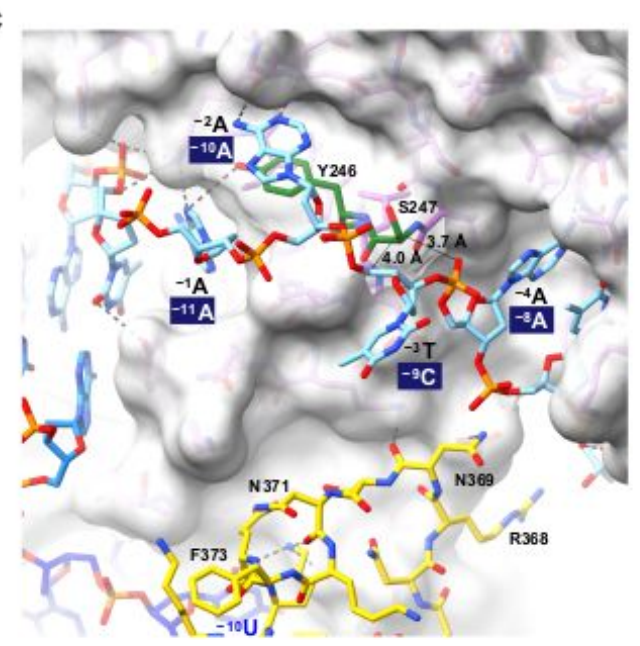

e

gp226 CTD WT

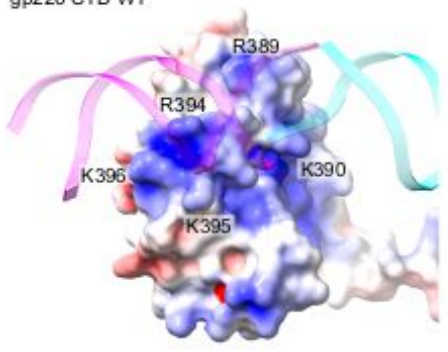

b

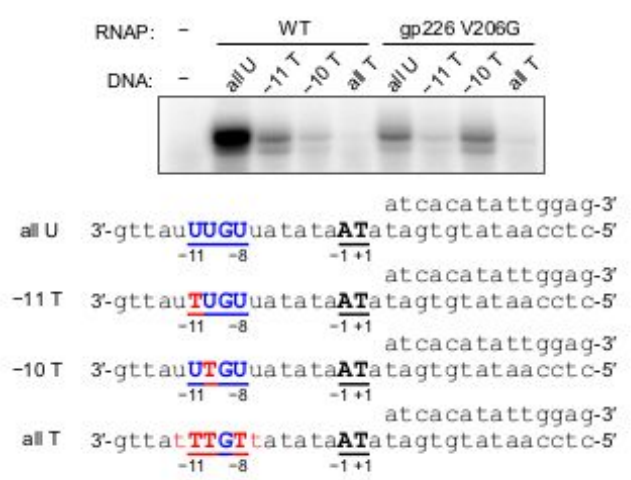

d

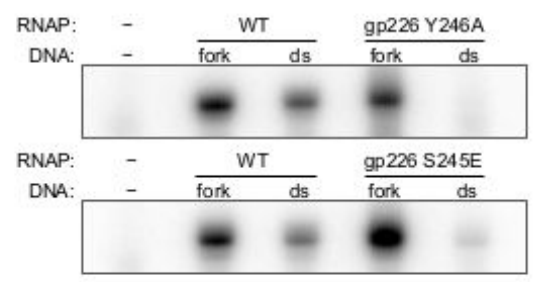

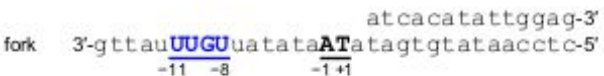

5'-caa taAACAa ta ta $t$ TAt at cacat at tggag-3'

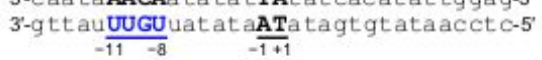

f

gp226 CTD R3asaK390A F394A K305A KOSSA

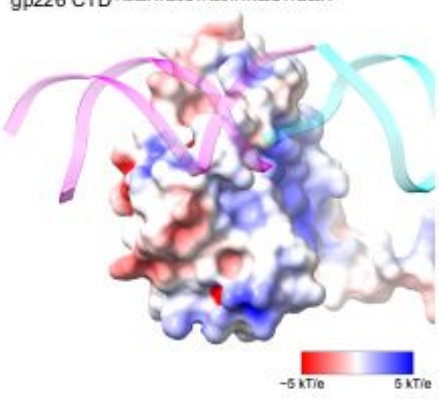

Figure 8

\section{g}

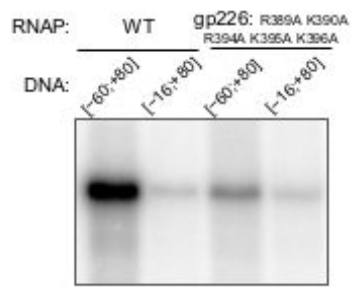

Figure 8 
Interaction of the AR9 nvRNAP with DNA in the promoter complex. a, Atomic model of the AR9 nvRNAP promoter recognition element. Gp226 is shown as a semitransparent molecular surface. Only a small fragment of $\beta \mathrm{N}$ gp105 that participates in the formation of the $-10 \mathrm{U}$ binding pocket is shown for clarity. Interchain and DNA-intrachain hydrogen bonds are shown as dashed lines. Thin, straight lines connect the $\mathrm{C} 5$ atom of the uracil pyrimidine ring to the closest protein or DNA atoms which lie in-plane with the ring. The carbon atoms are colored as in Fig. 2a, except for V206, which is shown in cyan. b, The in vitro transcription activity of the AR9 nvRNAP holoenzyme containing wild type (WT) gp226 or the V206G gp226 mutant have been tested using various U- and T-containing templates. c, Interaction of the upstream oligonucleotide with the gp226 NTD. The same gp226 and $\beta N$ gp105 fragments as in Fig. 8a are shown but are tilted to improve visibility. The nucleotides are numbered according to the original nomenclature of the template strand (see Fig. 2b). The putative base identities and their numbers relative to the TSS (as would be found in a dsDNA transcription bubble) are given in midnight blue colored boxes. $\mathrm{d}$, The in vitro transcription activity of the AR9 nvRNAP holoenzyme containing gp226 mutants with an altered structure of the non-template strand binding groove. e and $f$, The surface electrostatic potentials of the pseudo-35-element binding motif in WT gp226 and the A5 gp226 mutant. g, The ssDNA and dsDNA in vitro transcription activities of the AR9 nvRNAP holoenzyme containing WT gp226 and the A5 gp226 mutant. For each in vitro transcription experiment, two technical replicates of two biological replicates resulted in similar outcomes and one of them is shown. The uncropped autoradiograms are presented in Supplementary Fig. 5. 
a
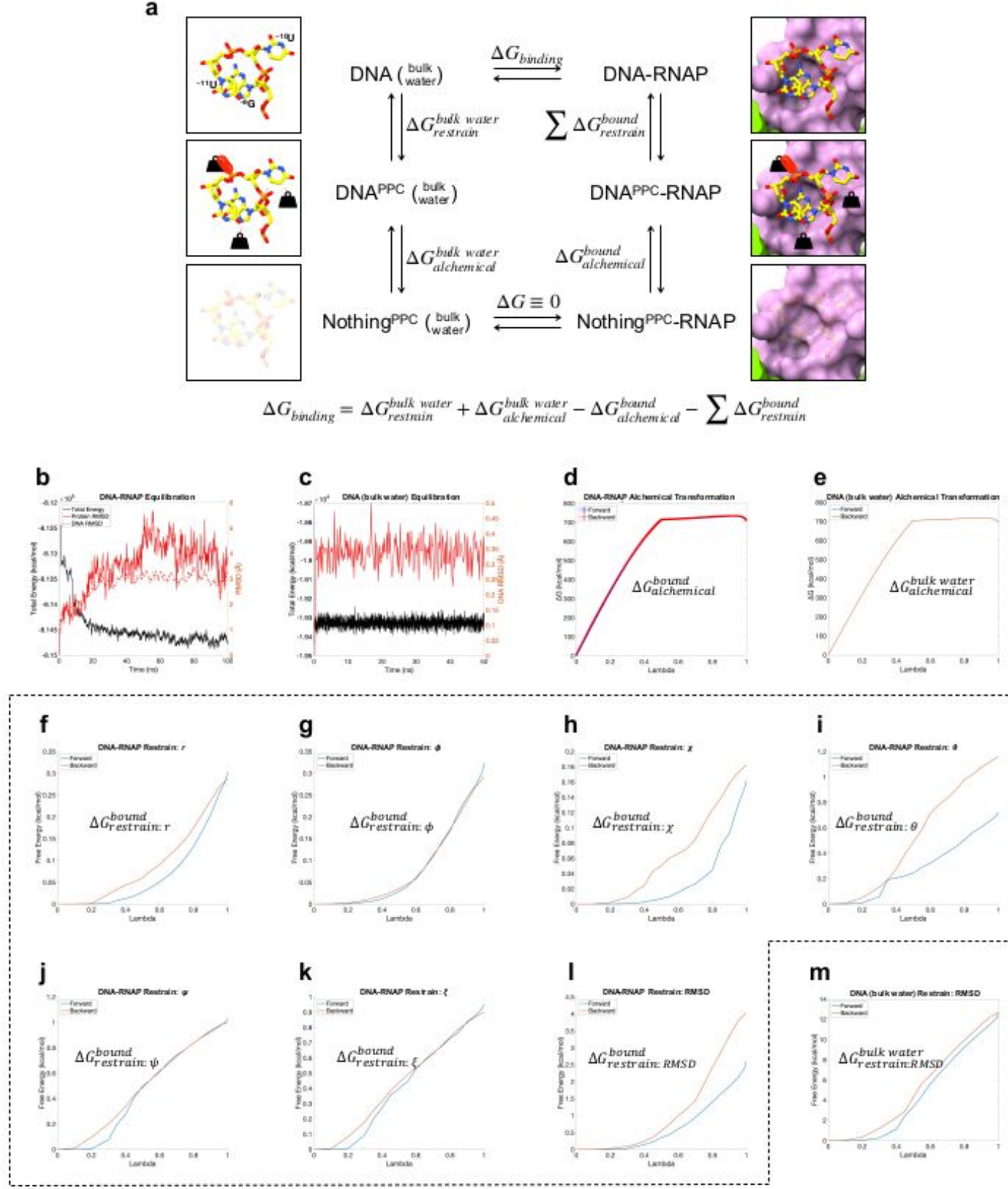

Figure 9

\section{Figure 9}

Derivation of the promoter binding free energy using molecular dynamics. a, Thermodynamic cycle of promoter binding. The PPC superscript (e.g. DNAPPC) stands for Promoter Pocket Conformation in regard to the structure of the 3'--11UUG-9-5' DNA trinucleotide. b, Equilibration and relaxation of the cryoEM derived atomic model of the AR9 nvRNAP holoenzyme with the 3'--11UUG-9-5' DNA trinucleotide bound to the promoter pocket. c, Equilibration and relaxation of the 3'--11UUG-9-5' trinucleotide in bulk 
water. $d$ and e, Energetics of forward and backward alchemical transformations of the 3'--11UUG-9-5' DNA trinucleotide in the promoter pocket of the AR9 nvRNAP holoenzyme and in the PPC in bulk water, respectively. f, g, h, i, j, k, I, Entropic cost of applying seven harmonic constraints to the 3'--11UUG-9-5' DNA trinucleotide to maintain it in the promoter pocket-bound state. $\mathrm{m}$, Entropic cost of the harmonic RMSD constraint on the 3'--11UUG-9-5' DNA trinucleotide to maintain the PPC.
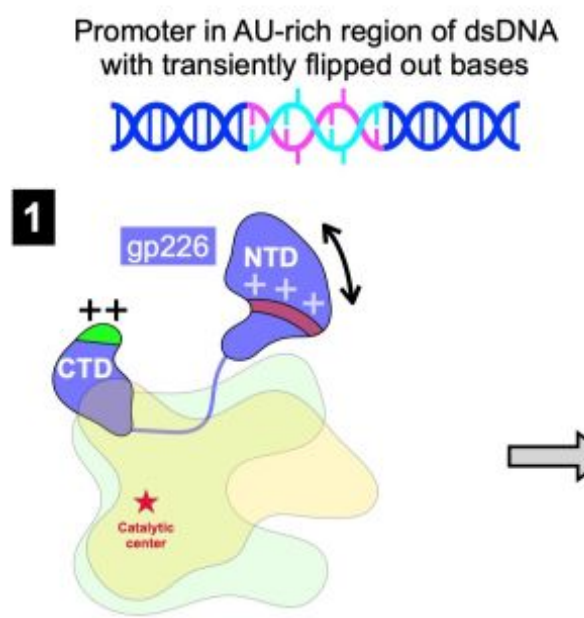

Enzyme is free in solution; gp226 NTD does not interact with the body of the enzyme.
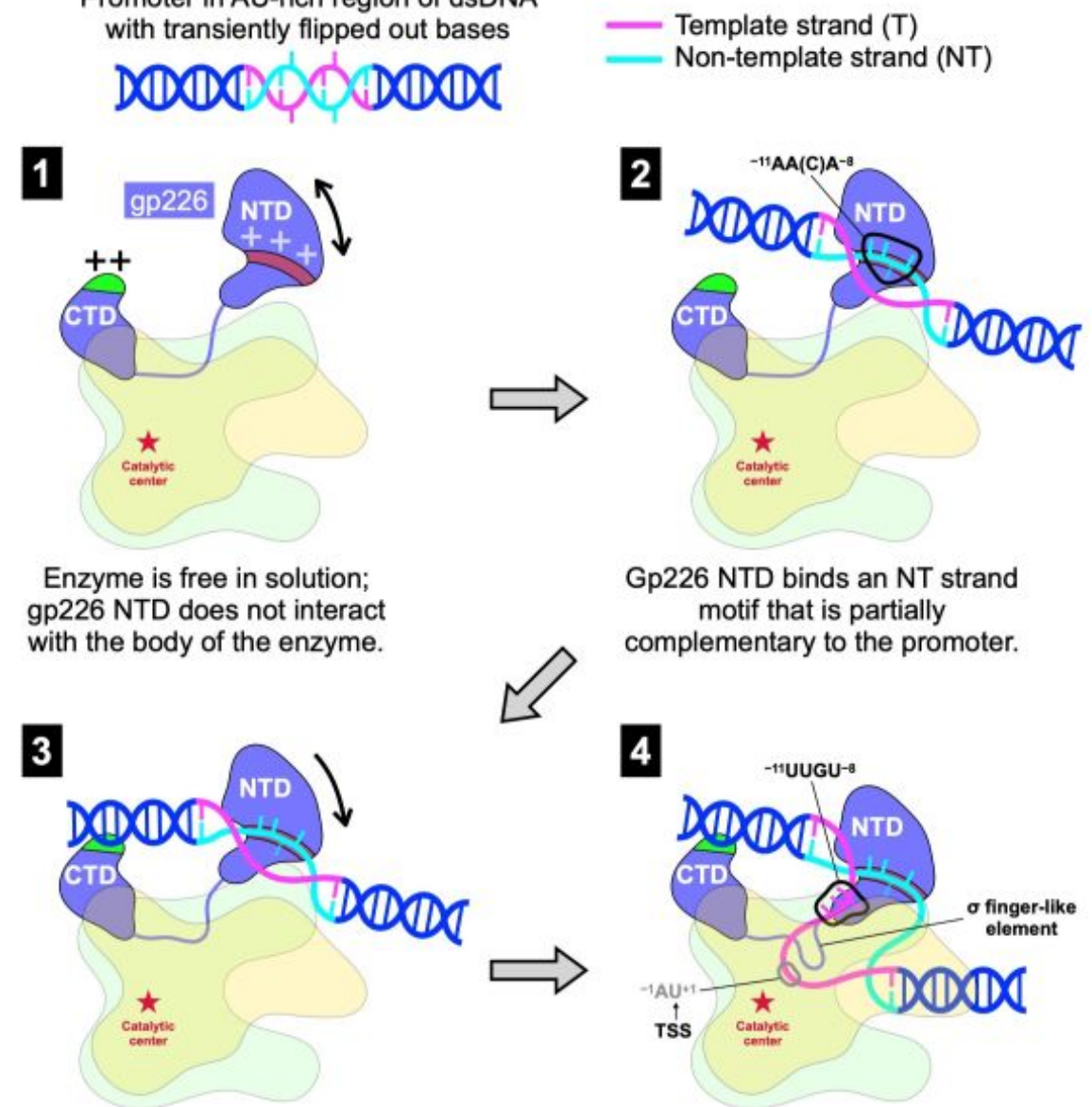

Gp226 CTD binds -35 region of dsDNA; mobility of gp226 NTD is reduced.

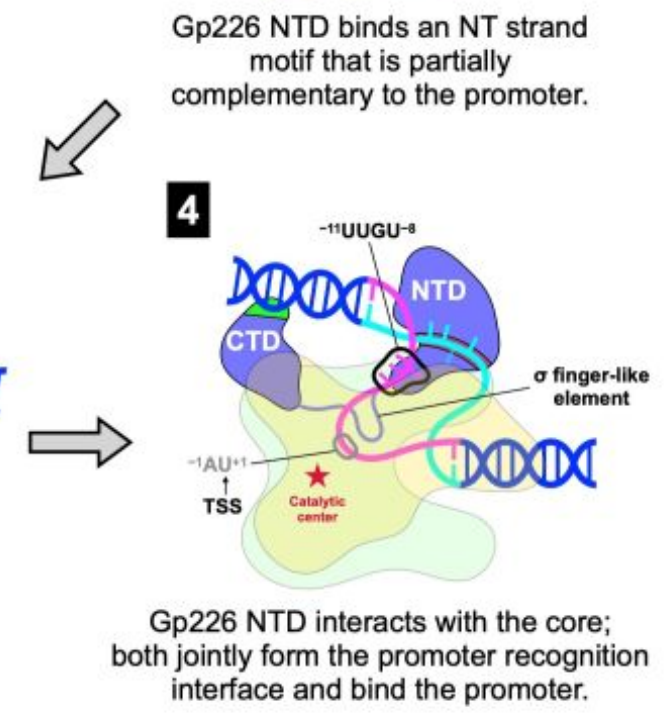

Figure 10 
The mechanism of template strand promoter recognition in dsDNA. For clarity, both proteins comprising the $\mathrm{N}$ - and $\mathrm{C}$-terminal parts of the $\beta$ and $\beta$ ' subunits are shown in the same color (light yellow for $\beta$ and light green for $\beta^{\prime}$ ). The putatively degenerate, third position base $-9 \mathrm{C}$ of the promoter-complementary motif $-11 A A(C) A-8$ is shown as a semitransparent rod. The pluses indicate positively charged elements of the gp226 NTD and CTD molecular surface that participate in DNA binding. See the main text for full explanation.

\section{Supplementary Files}

This is a list of supplementary files associated with this preprint. Click to download.

- NCOMMS2142281TRS.pdf

- SupplementaryInformation.pdf 